\title{
Zur Komorbidität von Posttraumatischer Belastungsstörung und Sucht in biopsychosozialer Perspektive
}

\author{
Hans-Peter Kapfhammer (D)
}

Eingegangen: 17. August 2020 / Angenommen: 16. Dezember 2020 / Online publiziert: 13. Januar 2021

(c) Der/die Autor(en) 2021

\begin{abstract}
Zusammenfassung Posttraumatische Belastungsstörung und Substanzkonsumstörungen treten im medizinischen Versorgungssystem häufig koexistent auf. Ihre Komorbidität geht mit schwerwiegenderen akuten klinischen Symptombildern, mit zahlreichen, oft notfallmäßigen Hospitalisierungen und geringeren Behandlungserfolgen einher. Ihre Komorbidität trägt zu dramatisch ungünstigeren Verläufen auf allen biopsychosozialen Ebenen bei. Das Thema Komorbidität von PTBS und Sucht wird auf mehreren Ebenen untersucht: in den Perspektiven von Epidemiologie, Substanzkonsumstörung als Risikofaktor für Trauma und PTBS, Trauma und PTBS als Risikofaktor für Substanzkonsumstörung, neurobiologischen Konsequenzen einer Substanzkonsumstörung für die Neurobiologie von PTBS, gemeinsam geteilten Faktoren der Genetik/Epigenetik, Persönlichkeitsdimensionen und aversiven/traumatogenen Einflüssen in der frühen Entwicklung. Der Hauptfokus der Analyse liegt auf den wechselseitig sich verstärkenden Mechanismen, die der Entwicklung und dem Verlauf beider Störungsbilder inhärent sind.
\end{abstract}

Schlüsselwörter Trauma P Posttraumatische Belastungsstörung · Substanzkonsumstörung · Komorbidität · Epidemiologie · Psychologie · Neurobiologie $\cdot$ Bindung

Herrn Univ. Prof. Dr.med. Michael Musalek zu seiner Emeritierung in freundschaftlicher Verbundenheit gewidmet.

Univ. Prof. Dr. med. Dr. phil. H.-P. Kapfhammer ( $\bowtie)$ Universitätsklinik für Psychiatrie und Psychotherapeutische Medizin, Medizinische Universität Graz, Auenbruggerplatz 31, 8036 Graz, Österreich hans-peter.kapfhammer@klinikum-graz.at

\section{Comorbidity of posttraumatic stress disorder and addiction from a biopsychosocial perspective}

Summary Posttraumatic stress disorder and substance use disorder often co-occur within the health care system. Their comorbidity is associated with more serious acute clinical symptomatology, more frequent hospital admissions in state of emergency and significantly lower chances of improvement by psychological and pharmacological treatment. Their comorbidity contributes to dramatically unfavourable courses of illness as regards all biopsychosocial levels. The survey presented will discuss empirical findings from various perspectives: general epidemiology, substance use disorder as risk factor of trauma and PTSD, trauma and PTSD as risk factor of SUD, neurobiological effects of SUD converging towards neurobiology of PTSD, shared common factors of genetics/ epigenetics, personality traits, and early developmental stress and trauma. The main focus of analysis will be put on processes that are intrinsically linked to the development and course of both disorders.

Keywords Trauma Posttraumatic stress disorder - Substance use disorder - Comorbidity . Epidemiology · Psychology · Neurobiology · Attachment

\section{Einleitung}

Posttraumatische Belastungsstörung (PTBS) und Substanzkonsumstörungen treten im Versorgungssystem häufig koexistent auf und können hier große diagnostische und therapeutische Herausforderungen bereiten. PTBS wie Sucht stellen in einer biopsychosozialen Perspektive gleichermaßen komplexe psychische 
Störungen dar, die es zunächst je für sich kurz zu skizzieren gilt.

Trauma impliziert im psychologischen Verständnis die Konfrontation mit einer unausweichlichen Bedrohung der eigenen leiblichen Existenz. Traumatische Erlebnisse verweisen auf Extremsituationen des menschlichen Lebens. Sie unterbrechen den Lauf einer individuellen Biographie abrupt. Im Falle einer kollektiven Betroffenheit verändern sie gleichzeitig das Leben zahlreicher Mitglieder einer sozialen Gruppe signifikant. Konsequenzen nach Traumata sind sehr vielfältig. Folgestörungen nach einem Trauma sind aber keineswegs normativ. In einer klinischen Perspektive sind viele Faktoren wie prätraumatische Vorbelastungen und psychiatrische Vorerkrankungen, quantitative wie qualitative Dimensionen des äußeren aktuellen Traumas, Attribution subjektiver Bedeutungen und psychobiologisches Coping in der persönlichen Lebens- und Entwicklungssituation zu beachten. Aber auch Einflüsse der posttraumatischen Umwelt wie Verfügbarkeit und Güte der interpersonalen und sozialen Unterstützung sind zu berücksichtigen, um Ausmaß und Vielfalt der posttraumatischen Reaktionen, die Fähigkeit zur psychologischen und psychosomatischen Erholung wie auch das Risiko für eine chronische Beeinträchtigung besser verstehen zu können.

Die Posttraumatische Belastungsstörung (PTBS) stellt die prototypische, aber nicht die einzige Traumafolgestörung dar. Eine PTBS zeichnet sich bis zu DSM-IV-TR und im ICD-10 durch drei Symptomkomplexe aus, durch intrusiv auftretende Erinnerungen an das Trauma, durch eine systematische Vermeidung aller Trauma-bezogenen Aspekte sowie durch zahlreiche körperliche und kognitive Symptome einer autonom-nervösen Überaktivität aus. In DSM-5 wird das klinische Bild einer PTBS breiter beschrieben. Es sind nunmehr auch negative Veränderungen in Kognitionen und Stimmungen in unmittelbarer Assoziation mit dem Trauma eigenständig abgebildet. Es werden hierüber dissoziative Erinnerungsstörungen, Depersonalisation/Derealisation und Gefühlsbetäubung, persistierende negative Überzeugungen oder Erwartungen an sich oder an die Umwelt, persistierende negative emotionale Zustände, Interessensverlust und Anhedonie erfasst. Eine der grundlegenden Erkenntnisse aus empirischen Studien ist, dass Traumata nicht nur dramatische psychologische Verarbeitungsprozesse auslösen, sondern auch tief in biologische Regulationssysteme eingreifen und funktionelle wie auch strukturelle Störungen im zentralen und autonomen Nervensystem nach sich ziehen können. Genetische Ausstattung, epigenetische Mechanismen und dispositionelle biologische Stresssysteme nehmen wesentlichen Einfluss darauf, ob eine spezielle traumatische Erfahrung $\mathrm{zu}$ schwerwiegenden psychischen Störungen (z.B. PTBS) und zu ernsten körperlichen Erkrankungsrisiken führt, oder aber mit einer erstaunlichen Kraft und Resilienz trotz anfänglicher Erschütterung gemeistert wird [92, 114].

Substanzkonsumstörungen werden heute ebenfalls als multifaktoriell bedingte, häufig chronisch verlaufende Störungsbilder definiert. Ein anthropologischer Blick auf „Sucht“ als existentielles Thema zwischen den Polen Rausch und Ekstase einerseits und Desintegration, Vereinsamung, Verzweiflung, Angst und Scham andererseits vermittelt wichtige Einsichten in das Grunddilemma eines süchtigen Menschen. Für ein tieferes klinisches Verständnis von Substanzbezogenen und nicht-stoffgebundenen „Süchten“ in ihrer Heterogenität und Komplexität reicht dieser Zugang aber allein nicht aus. Wesentliche Voraussetzung für eine integrative Perspektive ist, „Süchte“ als eigenständige Krankheiten zu begreifen. Je unterschiedliche biologische, psychologische und soziale Einflüsse begründen eine individuelle Vulnerabilität. Diese Vulnerabilität manifestiert sich in akuten Konsumepisoden unter speziellen Entwicklungs- und belastenden Lebensbedingungen. Wiederum andere Faktoren können einen chronischen Verlauf fördern. Grundlegend ist: Suchterkrankungen führen häufig zu dramatischen nachteiligen Konsequenzen auf allen Ebenen des persönlichen, familiären und sozialen Lebens. Folgen aus Suchterkrankungen schließen zahlreiche psychische und somatische Komorbiditäten, den Verlust tragender psychosozialer Rollen, häufig einen sozialen Abstieg und vorzeitigen Tod mit ein [180].

Nachfolgend soll das Thema Koexistenz/Komorbidität von Trauma-bezogenen Störungen und Störungen durch Substanzkonsum aufgenommen werden. Ein spezieller Fokus soll hierbei auf mögliche inhärente Zusammenhänge von PTBS und Sucht gerichtet sein. Grundsätzlich sind hierbei mehrere Bedingungskonstellationen ins Auge zu fassen:

- Zufällige Koinzidenz von Substanzkonsumstörung und PTBS

- Substanzkonsumstörung versursacht direkt oder indirekt PTBS

- PTBS versursacht Substanzkonsumstörung

- Gemeinsame Ursachen für Substanzkonsumstörung und PTBS

- Substanzkonsumstörung und PTBS verursachen sich wechselseitig und interagieren nachteilig im weiteren Verlauf

Diese Zusammenhänge sollen nachfolgend aus mehreren Perspektiven anhand empirischer Befunde dargestellt und diskutiert werden.

\section{Epidemiologische Perspektive}

Bereits erste epidemiologische Untersuchungen (Epidemiologic Catchment Area Studies) zeigten einen engen Zusammenhang von PTBS und Substanzkonsumstörungen in der Allgemeinbevölkerung, sowie besonders eindrucksvoll in störungsorientierten 
Behandlungskontexten. So bewegten sich die Prozentsätze von koexistenter Suchtstörung (Alkohol, Drogen) bei ehemaligen Angehörigen der US-Army mit der Diagnose einer PTBS in einem Bereich von 50-75\% [109]. Und umgekehrt wiesen Personen, die sich in Entwöhnungsprogrammen wegen Alkoholoder Drogenproblemen befanden, in über $40 \%$ auch eine zusätzliche PTBS-Diagnose auf [39]. Breit angelegte nachfolgende epidemiologische Surveys bestätigten diese signifikanten Assoziationen eindrucksvoll [151]. Sie spezifizierten auch nach einzelnen Substanzen.

In der für die US-amerikanische Bevölkerung repräsentativen Studie NESARC (National Epidemiological Survey of Alcohol and Related Conditions: $N=34.160$ ) betrug die Lebenszeitprävalenz für PTBSallein, Alkoholabhängigkeit-allein und Koexistenz von PTBS und Alkoholabhängigkeit je 4,83\%, 13,66\% und 1,59\%. Die Komorbiditätsgruppe zeichnete sich im Vergleich zu den beiden anderen Gruppen durch höhere Raten an frühkindlichen Traumatisierungen, durch häufigere Achse-I- und -II-Störungen, vermehrte Suizidversuche, einen früheren Beginn der PTBS, höhere PTBS-Symptomenanzahl, mehr Kriterien der Alkoholabhängigkeit, einen stärkeren zusätzlichen Drogenkonsum und gravierendere psychosoziale Behinderungsgrade aus [21]. In einer Detailstudie ebenfalls an diesem NESARC-Sample wurden Personen mit Alkohol-Missbrauch/Abhängigkeit sowie mit versus ohne PTBS hinsichtlich der prädiktiven Wertigkeit berichteter traumatischer Lebensereignisse ( $n=27$ distinkte Trauma-Typen) für eine inzidente Alkoholstörung untersucht. In einer multiplen Regressionsanalyse stellten sich für die Gruppe mit koexistenter PTBS statistisch signifikant erhöhte prädiktive Odds Ratios für die Traumatypen ,frühkindliches Trauma“ $(\mathrm{OR}=1,40 ; 1,08-1,83 ; p<0,01)$ und „gewaltsamer Übergriff" (OR=1,41; 1,13-1,77; $p<0,01)$, aber auch für die Patientengruppe ohne PTBS signifikant erhöhte ORs für "frühkindliches Trauma“ $(\mathrm{OR}=1,32 ; 1,23-1,41 ; p<0,001)$, „gewaltsamer Übergriff" $(\mathrm{OR}=1,42 ; 1,13-1,78 ; p<0,001)$, „unerwarteter Tod eines nahen Angehörigen" (OR=1,19; 1,12-1,28; $p<0,001)$ sowie „Mitteilung eines Traumas bei einem nahen Angehörigen" (OR=1,22; 1,13-1,30, $p<0,001)$ dar [61]. Systematische Reviews bekräftigten die enge Assoziation von Trauma, PTBS und Alkoholkonsumstörung sowohl für verschiedene Gruppierungen aus der Allgemeinbevölkerung als auch für die speziellen Populationen von Soldaten im aktiven Militärdienst und von Veteranen [44, 50, 74].

Systematische Reviews und Metaanalysen unterstrichen auch eine ähnlich starke Assoziation zwischen Trauma und PTBS einerseits und koexistentem Tabakkonsum (Zigarettenrauchen) andererseits. So betrug die durchschnittliche Prävalenz eines aktuellen Tabakkonsums bei Personen mit PTBS $24 \%$. Umgekehrt lag die koexistente Diagnose einer PTBS bei Zigarettenrauchern in $20,2 \%$ vor. Personen mit
PTBS zeichneten sich zudem durch eine hochgradige Nikotinabhängigkeit und einen massiven aktuellen Konsum von Tabakprodukten aus [146]. Verglichen mit nichtrauchenden Personen aus der Allgemeinbevölkerung zeigten rauchende Personen eine ca. doppelt so hohe Rate an koexistenter PTBS. An PTBS leidende Raucher waren im Vergleich $\mathrm{zu}$ Rauchern ohne PTBS durch eine höhere negative Affektivität, eine umfangreichere Trauma-Anamnese, eine stärker ausgeprägte Nikotinabhängigkeit, häufigere andere psychiatrische Komorbiditäten, eine höhere Anzahl frustraner Abstinenzversuche und vermehrte Rückfälle nach zuvor schon erreichter Abstinenz von Nikotin charakterisiert [95].

Auch für die Opiatabhängigkeit betonten epidemiologische Untersuchungen einen überzufällig engen Zusammenhang zu Trauma und PTBS. Während Surveys für die US-amerikanische Allgemeinbevölkerung in ca. $60 \%$ der Personen mindestens ein schwerwiegendes Trauma und eine durchschnittliche PTBS-Häufigkeit von $7 \%$ in der Lebenszeitspanne aufdeckten, lag die Prävalenz von bedeutsamen Traumata bei opiatabhängigen Personen über $90 \%$, die in ca. $40 \%$ der Fälle mit einer koexistenten PTBS einhergingen [128]. In der repräsentativen NESARCStudie betrugen diese Prävalenzraten von PTBS und Opiatkonsumstörung je für sich 4,7\% bzw. 4,3\%. Hinsichtlich einer Komorbidität waren drei Befunde herauszuheben: Die vielfach adjustierte Odds Ratio bei Personen mit PTBS im Vergleich zu Personen ohne PTBS bezüglich koexistenter Opiatkonsumstörung war mit adj. AOR=1,8 statistisch signifikant erhöht. Unter den Personen mit Opiatkonsumstörung (4,3\%) wiesen nur 0,7\% keine koexistente PTBS auf. Der Einfluss von chronischen Schmerzsyndromen bei PTBSPersonen war als signifikante Mediatorvariable für ein koexistentes Opiatkonsumrisiko besonders hervorzuheben (Muskelschmerzen: $31 \%$ - adj. OR=4,2; $p<0,001$; neuropathische Schmerzen: $25,2 \%$ - adj. $\mathrm{OR}=3,1 ; p<0,001$; abdominelle Schmerzen: 12,8\% adj. $\mathrm{OR}=1,3$; n.s. [18]). Eine Koexistenz von PTBS und Opiatkonsumstörung prädizierte mehrheitlich einen höheren Schweregrad des Krankheitsverlaufs sowie bedeutsamere Einbußen in der Gesundheitsbezogenen Lebensqualität [58, 73].

In einem epidemiologischen Fokus auf suchtspezifische Behandlungseinrichtungen stellte sich für Patient*innen mit zusätzlicher Diagnose PTBS/ Depression auch eine überproportional erhöhte Assoziation mit einem Mehrfachsubstanzkonsum dar $[32,113]$.

Epidemiologische Analysen der zeitlichen Abfolge der koexistenten Störungen hoben mehrheitlich eine vorausgehende PTBS und eine nachfolgende inzidente Substanzkonsumstörung hervor [30, 31, 46, 199]. Aber auch die umgekehrte zeitliche Sequenz von vorausgehender Substanzkonsumstörung und nachfolgender inzidenter PTBS wurde in einigen Studien angedeutet [44, 50, 87, 176]. Die Assoziation zwischen 
Trauma und erhöhtem Substanzkonsumrisiko schien vorrangig über den diagnostischen Status einer PTBS vermittelt zu werden, konnte aber nach bedeutsamen, vor allem kumulativen Traumata auch ohne inzidente PTBS auftreten ([9, 30, 47, 61, 114, 152, 199].

Auf den prägenden nachteiligen Einfluss von Traumata in frühen Entwicklungsabschnitten nicht nur für zahlreiche psychische, Verhaltens- und Substanzkonsumstörungen, sondern auch für eine Reihe von somatischen Erkrankungsrisiken im Erwachsenenalter ist gesondert hinzuweisen. Mehrere prospektive Longitudinaluntersuchungen haben diesen Befund nachhaltig bestätigt. Ein systematisches Review mit Metaanalyse fand für Personen mit $\geq 4$ aversiven oder traumatischen Belastungen in der Kindheit (ACEs) im Vergleich zu Personen ohne ACEs durchgängig signifikant erhöhte Risiken für alle untersuchten psychischen Störungen und somatischen Krankheiten im Erwachsenenalter. Die einzelnen Odds-Ratios stellten sich differentiell dar [82]:

- OR: <2: körperliche Inaktivität, Übergewicht, Adipositas, Diabetes mellitus

- OR: 2-3: Rauchen, bedeutsamer Alkoholkonsum, schlechter selbst-beurteilter Gesundheitszustand, Karzinome, Herzkrankheit, respiratorische Krankheit

- OR: 3-6: riskantes Sexualverhalten, psychische Morbiditäten (Depression, Angst, PTBS), problematischer Alkoholkonsum

- OR: >7: problematischer Drogenkonsum, selbstverletzendes Verhalten/Suizidalität, interpersonale Aggressivität/Gewalt

Mehrere Studien replizierten diese signifikante pathogenetische Rolle von frühkindlichen TraumaExpositionen für die Vermittlung einer Koexistenz/ Komorbidität von PTBS und spezieller Substanzkonsumstörung im Erwachsenenalter [61, 113, 115, 130, 156, 214]. Diese engen Assoziationen dürfen jedoch nicht in einem Modell linearer Kausalrelationen konzeptualisiert werden, sondern verweisen stets auf komplex vermittelte intermediäre Risikozustände, die schließlich in manifeste Störungsbilder einmünden können [93, 168].

Zusammenfassend betonen epidemiologische Studien einhellig, dass eine Koexistenz/Komorbidität von PTBS und Substanzkonsumstörung mit einem schwerwiegenderen klinischen Symptombild in akuten Krankheitsepisoden, mit häufigeren stationären Notfallaufnahmen, reduzierten Ansprechraten auf durchgeführte Behandlungen, einem schwierigeren und komplikationsreicheren Krankheitsverlauf, mit höheren interpersonalen Problemen, massiveren psychosozialen Behinderungsgraden und niedrigerer Gesundheits-bezogener Lebensqualität einhergeht. Eine bedeutsamere Suizidalität und ein erhöhtes Gesamtmortalitätsrisiko sind besonders zu beachten [63, 121, 138].

\section{Substanzkonsumstörung als Risikofaktor für Trauma und PTBS}

Eine von Substanzabusus bestimmte Lebenspraxis birgt per se ein erhöhtes Risiko für eine Reihe von möglichen Trauma-Expositionen. In diesem Fokus darf ebenfalls keine lineare Kausalversursachung aus der zeitlichen Abfolge von Substanzabusus, Traumaerfahrung und PTBS abgeleitet werden. In einer biopsychosozialen Perspektive ist zunächst grundlegend, dass soziale Kontexte, in denen Personen aufwachsen und leben, sowohl eine Reihe von gebündelten Traumata und chronischen Stressoren mit erhöhten Risiken für eine PTBS als auch gleichzeitig für Substanzkonsumstörungen vermitteln können [35, 82, 173]. In einem Substanzabusus und Traumatisierung bahnenden „Envirom“ üben die allgemeinen sozialen Determinanten von Gesundheit und Krankheit (sozialer Gradient, Stressoren/Traumata, frühe Entwicklung und Beziehungssicherheit, Erziehungs- und Ausbildungsqualität, soziale Exklusion, Diskrimination und Armut, Kontrolle über Arbeit, Jobsicherheit und Arbeitslosigkeit, soziale Unterstützung und $\mathrm{Zu}$ gang zum Gesundheitssystem, Gebrauch von Suchtstiftenden Substanzen, Qualität von Ernährung und Essverhalten) einen grundlegenden und anhaltenden Einfluss aus [120]. Hierbei ist nicht nur ein „Zuviel“ an Risikofaktoren, sondern in der Regel auch ein „Zuwenig“ an protektiven Faktoren bedeutsam [183]. Einige spezielle Aspekte eines krisenhaft entgleitenden oder chronischen Substanzkonsums, die inhärent eine vermehrte Exposition gegenüber Traumata und damit assoziiert ein erhöhtes PTBS-Risiko vermitteln, können zudem aufgezeigt werden:

- Hohe Raten an interpersonaler Gewalt, sexueller Ausbeutung und lebensgefährlichen Verletzungen charakterisieren eine traumatisierende soziale Lebenswelt zahlreicher drogenabhängiger Personen in ihrer alltäglichen Beschaffung von Substanzen [75, 86, 87, 169].

- Substanzmissbrauch und interpersonale Gewalterfahrungen definieren ein hoch komplexes Risikobündel speziell für Substanz-abhängige Frauen [3, 36].

- Der Zusammenhang von Substanzabusus (z.B. Rauschtrinken, „binge drinking“, Erstkonsum von illegalen Drogen) und erhöhtes Traumaexpositionsrisiko stellt in Adoleszenz und jungem Erwachsenenalter eine besondere entwicklungspsychopathologische Herausforderung dar [49, 60, 110, 144].

- Alkohol- und Drogen-induzierte Intoxikationen (v.a. beinah-letale Überdosierungen von Opiaten) implizieren unter speziellen körperlichen und psychosozialen Bedingungen ein hohes Risiko an gefährlichen Verletzungen, traumatisch erlebten Reanimationen, invasiv-chirurgischen und intensivmedizinischen Behandlungen. Sie alle können peritraumatische Symptome sowie in Folge auch eine 
posttraumatische Verarbeitung anstoßen [9, 37, 40, 88, 116].

Zusammenfassend sind für die Relation von Substanzkonsumstörung und Trauma/PTBS empirisch zahlreiche intermittierende Variablen $\mathrm{zu}$ beachten, die ein komplex interagierendes Bedingungsgefüge aufspannen. Substanzabusus im Kontext von Armut, Viktimisierung, sozial devianten Lebensverhältnissen führt zu kumulativen Risiken für Trauma und PTBS, die wiederum im Sinne einer eskalierenden Spirale den Substanzkonsum verstärken. Einige spezielle traumatogene Aspekte, die einem Leben mit chronischem Substanzkonsum oft inhärent sind, müssen hierbei beachtet werden. Zudem sind differentielle pharmakologische Effekte eines Substanzkonsums zu diskutieren, die auf neurobiologischer Ebene auch ein erhöhtes PTBS-Risiko nach einer Traumatisierung beinhalten (siehe unten).

\section{Trauma/PTBS als Risikofaktor für Substanzkonsumstörung - Selbstmedikation bei PTBS-Symptomen}

Eine klinisch attraktive Hypothese (Selbstmedikation) besagt, dass Personen mit schwerwiegenden psychischen Störungen, wie beispielsweise PTBS bestimmte psychotrope Substanzen mit dem subjektiven Ziel vermehrt einsetzen, um definierte PTBSSymptome und einen hiermit assoziierten emotionalen Distress zu lindern oder aus dem PTBS-Verlauf resultierende psychosoziale Stressoren besser zu kupieren. Ein verstärkter Substanzkonsum wird in dieser Sichtweise als eine Variante der Selbstmedikation in Ermangelung einer effizienteren Coping-Strategie verstanden [97].

Empirische Belege für diese Hypothese stammen zunächst aus zahlreichen epidemiologischen Studien, die nach schwerwiegenden Traumatisierungen eine nahezu parallele Entwicklung zwischen der Intensität der PTBS-Symptome und dem Ausmaß des jeweils unterhaltenen Substanzkonsums (Alkohol, Nikotin, Benzodiazepine, Opiate, Cannabis) im weiteren Verlauf abbildeten [26, 29, 56, 70, 78, 206]. Ergebnisse aus Langzeitstudien, die eine häufigere inzidente Substanzkonsumstörung nach Trauma und PTBS, nicht aber nach Trauma-Exposition alleine hervorhoben, gehen mit dieser Sichtweise ebenfalls gut konkordant [31, 158]. Belege für eine Selbstmedikationshypothese erbrachten auch Detailstudien, die einen erhöhten Substanzkonsum in Abhängigkeit von definierten PTBS-Symptomclustern (Hyperarousal, Vermeidung, intrusive Wiedererinnerung; negative Trauma-assoziierte Stimmungen und Kognitionen) als Strategien einer Selbstregulation analysierten [29, 57, 62, 80, 124, 172, 201]. Generell stellte sich ein signifikanter Zusammenhang zwischen dem Gesamt-Score über alle PTBS-Symptomcluster und der Intensität des berichteten Cravings für die untersuchten Substanzen
(Alkohol, Stimulantien, Opiate) dar. Es zeichneten sich bei bevorzugt eingesetzten Substanzen (,drug of choice") einige typische Assoziationen zu speziellen PTBS-Symptomclustern ab (Stimulantien: PTBS-Vermeidung; Alkohol: PTBS-Hyperarousal; Opiate: kein spezielles PTBS-Symptommuster [179]).

Eine Reihe von Studien konzentrierte sich auf Variablen, die den Zusammenhang von PTBS und koexistenter Substanzkonsumstörung vermitteln könnten. Personen mit PTBS setzen offenkundig Substanzen vor allem in Augenblicken heftiger, nur schwer kontrollierbarer Emotionen ein, um hierüber zu einer günstigeren Selbstkontrolle zu gelangen [28, 191, 192]. Im Kontext einer PTBS bezieht sich eine emotionale Regulationsstörung nicht nur auf massive negative Affektzustände. Sie kann auch bei starken positiven Emotionen aufgezeigt werden [204, 205]. Als wahrscheinlicher Pfad zu einem verstärkten koexistenten Substanzkonsum zeichnet sich ab: Heftige emotionale Zustände mit Bezug zu Traumaerinnerung und -verarbeitung überfordern die Regulationsfähigkeit einer Person. Sie gehen mit einer reduzierten Selbstwirksamkeit einher. Sie führen zu einem erhöhten subjektiven Distress und triggern eine Reihe von potenziell gesundheitsschädigenden Verhaltensweisen (z. B. riskantes Sexual-, aggressives Verhalten). Sie favorisieren auch den Einsatz einer psychotropen Selbstmedikation, die als Risikoverhalten für eine nachfolgende Substanzkonsumstörung gewertet werden muss $[1,155$, 157, 181, 203]. Das Persönlichkeitsmerkmal Impulsivität wird über die unmittelbar durch einen Trauma-Triggerreiz ausgelöste emotionale Antwort angezeigt. Impulsivität vermittelt als Co-Risikofaktor die Assoziation von PTBS-Symptomintensität und koexistentem Alkoholkonsum bedeutsam [119, 160]. Dieser Zusammenhang kann für andere Drogen aber nicht in vergleichbarer Weise bestätigt werden. Bei koexistenter Kokainabhängigkeit etwa wird die Aufrechterhaltung eines Konsums sehr viel stärker von einer sich bietenden situativen Gelegenheit relativ unabhängig vom PTBS-Status gesteuert [190, 198]. Die Relation von PTBS und koexistenter Alkoholkonsumstörung nach sexueller Traumatisierung wird insbesondere bei Frauen stark durch die kognitive Variable einer Selbstschuld-Attribution („self-blaming“) beeinflusst [99, 147]. Bei einer Vergewaltigung unter Alkoholeinfluss und nachfolgender PTBS sind zustandsabhängige intrusive Trauma-Erinnerungen unter erneutem Alkoholkonsum und eine zusätzlich verstärkte PTBS-Symptomatik in Folge zu bedenken [83].

Zusammenfassend lässt sich die epidemiologisch hohe Koexistenz/Komorbidität von PTBS und Substanzkonsumstörung teilweise auch als Folge eines vorrangig emotionsgesteuerten Copings bei PTBS verstehen, als Versuch mittels psychotroper Substanzen eine günstigere Selbst- und Emotionskontrolle zu erreichen. 


\section{Neurobiologische Konsequenzen von Substanzmissbrauch und -abhängigkeit in Konvergenz mit der Pathophysiologie der PTBS}

Alle psychotropen Substanzen, die ein Suchtpotential aufweisen, beeinflussen das natürliche Belohnungssystem entweder direkt oder indirekt durch einen vermehrten Dopamin-Turnover speziell in den limbischen Bahnen zwischen der Area tegmentalis ventralis des Mittelhirns und dem Nucleus accumbens (ventrales Striatum) der Basalganglien [135]. Das Ausmaß der dopaminergen Aktivierung an den $\mathrm{DA}_{2}$-Rezeptoren des Ncl. accumbens ist unmittelbar mit der Raschheit und Intensität der ausgelösten Belohnung korreliert. Das subjektive Genusserlebnis selbst wird aber nicht durch Dopamin, sondern vorrangig durch endogene Opioide, Endocannabinoide und GABA vermittelt [106]. Die Belohnungsvalenz einer bestimmten Substanz und des hierauf gerichteten Konsumverhaltens wird unter Einfluss von Amygdala und Hippokampus auch an den speziellen situativen Kontext des Konsums gebunden. Diese Lernschritte zur Motivation und Realisierung eines bestimmten Konsumverhaltens werden unter kognitiver, evaluativer und exekutiver Beteiligung des präfrontalen und motorischen Kortex wesentlich über Glutamat reguliert [90, 91]. Ein zentrales neurobiologisches Korrelat in der Entwicklung einer Substanzkonsumstörung stellt die molekulare Gegenregulation im Belohnungssystem selbst dar. Diese vollzieht sich vorrangig über eine verstärkte Aktivierung der к-Opiatrezeptoren durch Dynorphin. Sie führt $\mathrm{zu}$ einer Verminderung der belohnenden und analgetischen Effekte von endogenen Opioiden/ zugeführten Opiaten an den $\mu$ - und $\delta$-Opiatrezeptoren (Toleranzentwicklung). Andererseits kommt es über eine parallele Aktivierung der evolutionären Stresssysteme (HPA-Achse, autonomes Nervensystem, Immun-/Inflammationssystem; CRF, Kortisol, Nordrenalin/Adrenalin, Zytokine) $\mathrm{zu}$ einer weiteren Gegenregulierung. Diese Systeme induzieren eine erhöhte Stressanfälligkeit (Entzugssymptomatik) und intensivieren gleichzeitig ein verstärktes Suchverhalten nach belohnenden Substanzen. Das Grundmerkmal einer jeden Sucht, nämlich eine immer stärker eskalierende Diskrepanz zwischen einem sensitivierten Craving („wanting") einerseits und einem reduzierten Belohnungsempfinden (,liking“) andererseits, geht mit einer Umstellung der Verhaltensinitiierung von einem Modus der „positiven Verstärkung“ auf einen der „negativen Verstärkung“ einher [16, 17, 105]. Die Verhaltensausführung vollzieht sich hierbei unter einer bedeutsam eingeschränkten regulativen Funktionalität präfrontaler Strukturen [111].

In einer neurobiologischen Sicht auf die Koexistenz/Komorbidität von PTBS und Sucht ist grundlegend, dass beide Störungen prinzipiell innerhalb derselben neuronalen Regulationssysteme organisiert werden und gemeinsame molekulare Mechanismen teilen. Sie können sich in ihrem Verlauf wechselseitig verstärken [52, 53, 121, 184].

- Akuter Stress (z. B. bei Trauma-Exposition) bewirkt zunächst ganz ähnlich wie Substanzen mit suchtstiftendem Potenzial eine starke dopaminerge Aktivierung der Dopaminrezeptoren im Ncl. accumbens. Stress löst in weiterer Folge auch die skizzierte Kaskade einer vermehrten Freisetzung von endogenen Opioiden, Endocannabinoiden und GABA, aber auch von Glutamat aus. Hieran sind auch eine aktivierte HPA-Achse sowie ein erhöhter noradrenerger Turnover beteiligt.

- Chronischer Stress, wie paradigmatisch eine PTBS, mündet in einen vergleichbaren Reaktionszustand des Belohnungssystems ein, wie er für einen chronischen Alkohol- oder Drogenkonsum charakteristisch ist. Auch eine PTBS geht mit einem auffälligen Belohnungsdefizit im Nucleus accumbens einher [134]. Diese depressions-ähnliche Anhedonie wird nach DSM-5 im D-Cluster einer PTBS kodiert. Eine PTBS kann, ganz analog wie Substanzkonsumstörungen, auch mit prominenten kognitiven, evaluativen und exekutiven Funktionsdefiziten im präfrontalen Kortex assoziiert sein [38].

- Die sensitivierten Stresssysteme bei PTBS (v.a. sensitiviertes Noradrenalinsystem, dysfunktionale HPA-Achse) nehmen einen signifikanten Einfluss auf die Aufnahme und Intensivierung von suchtfördernden Substanzen. Sie erhöhen die Rückfallgefährdung nach intermittierend erreichter Abstinenz. Akute und chronische PTBS-Stressoren verstärken die Effekte von Substanz-bezogenen Schlüsselreizen auf das Dopaminsystem. Unter den Bedingungen der langfristigen neuroadaptiven Veränderungen des Belohnungssystems und der hiermit vernetzten kortikalen Kontrollstrukturen wird das inhärente Dilemma einer koexistenten Substanzkonsumstörung noch weiter eskaliert: Eine drangund letztlich zwangsbestimmte Verhaltensregulation in der Beschaffung psychotroper Substanzen (,wanting“; Glutamat, $\mathrm{DA}_{1}$-Rezeptoren) wird gebahnt. Gleichzeitig gelingt die Realisierung des angestrebten Genusseffektes (, liking“; reduzierte Ansprechbarkeit der $\mathrm{DA}_{2}$-Rezeptoren) immer weniger. Substanzen dienen vorrangig der vorübergehenden Beruhigung des induzierten emotionalen Distresses und der korrelierten physiologischen Stressreaktionen. Die Sucht-inhärente Stressaktivierung wirkt sich wiederum nachteilig intensivierend auf einzelne PTBS-Cluster (v. a. intrusive Traumaerinnerung, autonomes Hyperarousal) aus [54, 197].

Einzelne Substanzen mit Suchtpotential besitzen neben dem allgemeinen pharmakologischen Wirkprofil auf das Belohnungssystem auch einige differentielle Effekte, die im Kontext der Pathophysiologie der PTBS Relevanz besitzen: 
- Alkohol wird von PTBS-Patient*innen in seinem stark Distress-reduzierenden Effekt geschätzt und häufig für ein günstigeres Selbstmanagement eingesetzt. Alkohol beruhigt, entspannt, entängstigt und wirkt in steigenden Dosen Schlaf-anstoßend. Seine pharmakologische Hauptwirkung wird über den $\mathrm{GABA}_{\mathrm{A}}$-Rezeptor vermittelt, der $\mathrm{zu}$ einer Verschiebung in der Balance zwischen hemmendem GABA- und exzitatorischem Glutamat-System führt. Nach einer Trauma-Exposition zeichnen sich bei einem Übergang in eine PTBS stark reduzierte kortikale GABA-Spiegel (v.a. parietal, okzipital, anteriores Zingulum, Insel) bei gleichzeitig erhöhten Glutamat-Konzentrationen (v.a. temporal) ab. Die Schwere der PTBS-Symptomatik, insbesondere der Schlafstörungen ist hiermit korreliert [127, 162]. Alkoholkonsum kann dieses GABA-erge Defizit bei PTBS-Patienten zumindest vorübergehend mindern und auch die Intensität von aktuellen PTBSSymptomen abschwächen [145]. Alkohol mitigiert zwar eine Stress-induzierte Erhöhung des Cortisol- und Noradrenalin-Outputs, chronischer Alkoholkonsum oder starkes Rauschtrinken („binge drinking“) sind aber mit erhöhten tonischen Kortisol- und Noradrenalinspiegeln assoziiert [207]. Chronischer Alkoholkonsum verringert zudem die GABA-Rezeptoren und erhöht kompensatorisch die glutamatergen NMDA-Rezeptoren. Beide neurobiologische Anpassungen tragen $\mathrm{zu}$ einer StressSensitivierung bei, die sowohl die Alkoholkonsumstörung als auch die PTBS intensivieren und komplizieren $[5,76]$.

- Nikotin wird ähnlich häufig wie Alkohol von PTBSPatient*innen als Selbstmedikation im Umgang mit den posttraumatischen Symptomen verwendet [95]. Nikotin zeichnet sich durch ein polyvalentes Wirkprofil aus. Es wirkt gleichzeitig sedierend, anxiolytisch, antidepressiv, konzentrationsfördernd und allgemein stimulierend. Es entfaltet seine Wirkung über die präsynaptischen nikotinergen Acetylcholinrezeptoren, v. a. vom $\alpha_{4} \beta_{2}$-Subtyp. Es kommt dosis- und kontextabhängig hierüber zu einer erhöhten zerebralen Verfügbarkeit von Dopamin, Serotonin, $\beta$-Endorphinen, Noradrenalin, Acetylcholin und Vasopressin [10]. Bei chronischem Nikotinkonsum überwiegen aber die Stress-induzierenden Effekte einer aktivierten HPA-Achse mit vermehrter Sekretion von Kortisol und eines sensitivierten Noradrenalinsystems mit je nachteiligen Effekten sowohl auf den Verlauf der PTBS als auch der koexistenten Nikotin-Konsumstörung [207].

- Benzodiazepine zeigen mehrfache tranquilisierende Effekte (anxiolytisch, sedierend, schlafanstoßend, muskelrelaxierend, antiepileptisch). Sie entfalten eine agonistische Wirkung am $\mathrm{GABA}_{\mathrm{A}}$ Rezeptor des hemmenden GABA-Systems. Tierexperimentell kann über GABAerge Interneurone ein modulierend hemmender Effekt auf die Konsolidierung von aversiven/traumatischen Erinnerungen in den basolateralen Anteilen der Amygdala nachgewiesen werden $[118,161]$. Es ließe sich hieraus ableiten, dass nach einer Traumaexposition der prophylaktische Früheinsatz von Benzodiazepinen einer noradrenergen, aber auch glutamatergen Hyperaktivität entgegenwirken und eine überstarke Konsolidierung verhindern könnte. Die wenigen empirischen psychopharmakologischen Studien an Patientensamples bestätigten diese Hypothese aber nicht. Benzodiazepine waren im Vergleich zu Placebo nicht nur nicht überlegen, sie erhöhten nach einem Trauma sogar die Übergangswahrscheinlichkeit zu PTBS und Depression [65, 126]. Diese im klinischen Kontext potenziell nachteiligen Effekte von Benzodiazepinen im akuten Routineeinsatz nach einem Trauma werden mit einer dysmnestischen und dissoziativen Wirkung auf die Organisation des Trauma-Gedächtnisses und mit einer Behinderung des Extinktionslernens in Verbindung gebracht. Benzodiazepine zeigen ferner einen hemmenden Effekt auf die HPA-Achse und reduzieren die Sekretion von Kortisol [215]. Niedriges Kortisol wird aber als Risikofaktor für PTBS nach einem Trauma diskutiert [212]. Eine beeinträchtigte hemmende Funktion auf die intrusiven Traumaerinnerungen bei erniedrigtem Kortisol wird postuliert [154]. Benzodiazepine können die Symptomcluster bei einer etablierten PTBS nicht signifikant bessern [13]. Trotzdem sind die Verordnungszahlen/ Konsumraten von Benzodiazepinen bei PTBS-Patient*innen, vor allem bei Frauen nach wie vor sehr hoch [13, 15, 100]. Die nachteiligen Effekte eines chronischen Benzodiazepinkonsums sind in ihren neurobiologischen Auswirkungen auf die PTBS-Pathophysiologie ähnlich denen eines chronischen Alkoholkonsums zu bewerten (siehe oben).

- Cannabinoide wie Cannabis (psychoaktive Komponente: $\Delta_{1}$-Tetrahydrocannabinol, THC) oder Cannabidiol (CBD) entfalten ihre Effekte über die $\mathrm{CB}_{1}$ und $\mathrm{CB}_{2}$-Rezeptoren auf das Endocannabinoidsystem des Gehirns. THC wirkt an den $\mathrm{CB}_{1}$ - und $\mathrm{CB}_{2}$ Rezeptoren partiell agonistisch. CBD hingegen ist am $\mathrm{CB}_{1}$-Rezeptor antagonistisch und kann einen Teil der THC-Effekte aufheben. Cannabinoiden werden zahlreiche Effekte zugeordnet, von denen die Wirkungen bei chronischen Schmerzsyndromen, Übelkeit und Erbrechen bei Chemotherapie, Spastizität bei Multipler Sklerose noch die gesichertste empirische Evidenz besitzen [133]. Cannabisprodukte werden wegen ihrer emotional ausgleichenden Wirkung im Vergleich zur Allgemeinbevölkerung dreimal häufiger von PTBS-Patient*innen konsumiert [96]. Das hierüber aktivierte Endocannabinoidsystem nimmt einen modulierenden Einfluss auf die Konsolidierung des Gedächtnisses, den Retrieval-Prozess und die Extinktion von emotionalen und traumatischen Erfahrungen in den Kernregionen der basolateralen Amygdala, des Hippocampus und des präfrontalen Kortex. $\mathrm{CB}_{1}$-agonis- 
tische Cannabinoide wie z. B. THC stören den Retrievalprozess (Blockade der unwillkürlichen Traumaerinnerungen), fördern aber möglicherweise das Extinktionslernen [142]. Bei PTBS-Patient*innen waren die Konzentrationen des Endocannabinoids Anandamid erniedrigt, die Verfügbarkeit des $\mathrm{CB}_{1}$-Rezeptors kompensatorisch erhöht. Die $\mathrm{CB}_{1^{-}}$ Rezeptorverfügbarkeit in der Amygdala korrelierte positiv mit einer abnormen Prozessierung von Gefahrenreizen und einem erhöhten Niveau eines autonomen Arousal, aber nicht mit dysphorischen Symptomen [136]. Cannabiskonsum kann einige PTBS-Symptome abschwächen. Für das synthetische Cannabinoid Nabilone wurde in einem randomisierten, doppelblinden und placebokontrollierten Cross-over-Design eine gute Wirksamkeit bei PTBS-assoziierten Albträumen nachgewiesen [85]. Selektive Cannabinoide werden in der Behandlung der PTBS nach positiven Einzelfallstudien unter Beachtung der vorliegenden neurobiologischen Befunde zum Endocannabinoidsystem als eine weitere pharmakologische Therapievariante diskutiert [77]. Rezente systematische Reviews mit Metaanalyse hielten für den therapeutischen Einsatz von Cannabinoiden bei PTBS derzeit aber eine nur eine sehr geringe Evidenz für positive Effekte auf die PTBS-Symptomatik fest [19, 140]. Eine koexistente Cannabiskonsumstörung kann zudem zahlreiche PTBS-assoziierte Probleme wie kognitive und motivationale Defizite weiter verschärfen. Langfristig persistierende maladaptive Veränderungen in der HPA-Achse und im Noradrenalinsystem sind im Hinblick auf den PTBS-Verlauf nachteilig zu bewerten [207]. Die problematischen Effekte von Cannabis auf die Reifungs-abhängige zerebrale Reorganisation in Adoleszenz und jungem Erwachsenenalter sind besonders kritisch zu bedenken [72].

- Amphetaminerge Stimulantien und Kokain werden von einer Subgruppe von PTBS-Patient*innen ebenfalls überproportional häufig konsumiert [68, 164, 188]. Die euphorisierenden und allgemein aktivierenden Effekte werden über komplexe Mechanismen vermittelt. Ein pharmakologischer Hauptpfad hebt hervor: Kokain und Amphetamine sind kompetitive Substrate für Dopamin-, Noradrenalin- und Serotonin-Transporterproteine. Stimulantien gelangen statt der monoaminergen Neurotransmitter, die so vermehrt im synaptischen Spalt verbleiben, in das präsynaptische Terminal. Hier blockieren sie die Aufnahme der lokal synthetisierten Monoamine in die präsynaptischen Vesikeln durch eine Blockade der vesikulären Monoamintransporter $2\left(\mathrm{VMAT}_{2}\right)$ und führen $\mathrm{zu}$ einer verstärkten Freisetzung der Monoamine. Eine Blockade der Monoaminoxidasen bewirkt eine zusätzliche Anreicherung von Dopamin, Noradrenalin und Serotonin im synaptischen Spalt $[45,123]$. Amphetamine und Kokain können das PTBS-assoziierte Belohnungsdefizit vorübergehend wirksam antagonisieren, münden aber über den allgemeinen Sucht-stiftenden Circulus vitiosus sekundär in dieses ein und verstärken es signifikant [4]. Eine koexistente StimulantienKonsumstörung geht mit einer Intensivierung des Hyperarousal-Clusters einer PTBS einher und fördert wiederum einen verstärkten missbräuchlichen Beikonsum von tranquilisierenden Substanzen wie Alkohol, Benzodiazepinen, Opiaten oder Cannabis [51, 208]. Bedeutsame kognitive Defizite, aber auch erhöhte andere neuropsychiatrische, kardio- und zerebrovaskuläre Risiken sind bei einer Koexistenz mit PTBS zusätzlich zu berücksichtigen $[67,153]$.

- Das 3,4-Methylendioxy-N-Methylamphetamin (MDMA, Ecstasy) zeigt eine stimmungsaufhellende, Empathie und sozialen Kontakt fördernde Wirkung. MDMA erfreut sich einer großen Beliebtheit in der Partyszene. Die missbräuchliche Einnahme von MDMA zeigt ähnlich bedeutsame neuropsychiatrische Nebenwirkungen wie auch andere Stimulantien [194]. Für den PTBS-Verlauf ist eine koexistente MDMA-Konsumstörung vergleichbar problematisch einzustufen. Der pharmakologische Hauptwirkmechanismus von MDMA ist den klassischen Amphetaminen sehr ähnlich, die im ZNS verstärkte Freisetzung von Dopamin ist im Vergleich zu Noradrenalin und Serotonin aber geringer. Weitere neurobiologische Effekte sind eine Reduktion der amygdalären Hyperaktivität bei gleichzeitiger Aktivierung präfrontal-kortikaler Strukturen, eine Erhöhung der Oxytocin-Spiegel, eine positive Beeinflussung von Furchtextinktion und Gedächtniskonsolidierung. Diese speziellen Effekte werden derzeit hinsichtlich eines möglichen therapeutischen Potentials bei der PTBS diskutiert [108]. Erste kontrollierte Studien zur Augmentation von Trauma-Psychotherapien (z. B. prolongierte Exposition) mittels MDMA haben zu ermutigenden Ergebnissen geführt [7, 59, 84]. Angesichts noch nicht klar definierter Ausschlusskriterien, einer noch ungenügend erprobten Dosisfindung und weitgehend fehlender Erfahrungen in der Langzeitanwendung wird eine vorsichtige Zurückhaltung empfohlen [165]. Die vorliegende empirische Datenlage zur Wirksamkeit und Sicherheit von MDMA zur Augmentation von Trauma-Psychotherapien wird für eine FDA-Zulassung derzeit als noch nicht ausreichend beurteilt [159].

- Opiate werden gleichfalls von zahlreichen PTBS-Patient*innen, speziell mit assoziierten chronischen Schmerzsymptomen zur Selbstmedikation eingesetzt [143]. Zwischen einer Opiat-Konsumstörung, einem chronischen Schmerzsyndrom und einer PTBS bestehen große neurobiologische Parallelen. Alle drei klinischen Syndrome konvergieren in einem auffälligen „Belohnungsdefizit“, das sich durch eine verringerte Aktivierung der dopaminergen $\mathrm{DA}_{2 / 3}$-Rezeptoren im Ncl. accumbens (ventrales Striatum) darstellen lässt $[25,52,53]$. 
- Die Zusammenhänge von Trauma, PTBS und endogenes Opiatsystem sind komplex. Wichtige klinische Argumente für eine Beteiligung des Opiatsystems an der Pathophysiologie der PTBS sind [92]: Vor allem Frauen mit PTBS nach interpersonellen Gewalttraumata zeigen eine hohe Rate an chronischen Schmerzsyndromen. Personen mit sexuellem Missbrauch in ihrer frühen Entwicklung weisen eine signifikant erhöhte Rate an Opiatsubstanzmissbrauch auf. Eine der bedeutsamsten prophylaktischen pharmakologischen Maßnahmen nach schweren traumatischen Verletzungen und heftigen akuten Schmerzzuständen ist hinsichtlich eines späteren PTBS-Risikos die Gabe hoher Dosen von Morphinen. Entzugssymptome können nach einer prolongierten Stresssituation auftreten oder aber durch die Gabe des Opiatantagonisten (z. B. Naloxon) ausgelöst werden. Bei PTBS-Patient*innen finden sich im Liquor erhöhte Konzentrationen an $\beta$-Endorphinen.

- Endogene Opioide entfalten über ein heterogenes Muster an $\mu-, \delta-$, k-Opiatrezeptoren differenzielle Wirkungen [193]. Sie hemmen bei Trauma-Exposition die Schmerzwahrnehmung und reduzieren die v. a. noradrenerg getriggerten Panikaffekte. Die Amygdala ist besonders reich an Opiatrezeptoren. Eine über Opioide vermittelte psychomotorische Erstarrung und affektive Betäubung (,freezing/ numbing“) erlaubt möglicherweise dem Organismus, einen überwältigenden Stress nicht bei klarem Bewusstsein zu überstehen und auch die traumatische Erfahrung nicht exakt zu speichern. Eine negative Interferenz mit Lern- und Gedächtnisprozessen ist die Folge hoher endogener Opiatkonzentrationen. Ein differenzielles $\mu$-Opiatrezeptorbindungsverhalten stellte sich in einer PET-Studie bei Personen nach einer Traumaexposition mit und ohne nachfolgende PTBS dar. Beide traumaexponierten Gruppen unterschieden sich von gesunden Kontrollprobanden ohne Traumaerfahrung durch eine niedrigere Bindungskapazität in der erweiterten Amygdala, im Nucleus accumbens sowie im frontalen und dorsalen insulären Kortex und durch eine höhere Bindung im orbitofrontalen Kortex. PTBS-Patient*innen wiesen niedrigere Werte im anterioren Zingulum auf gegenüber den beiden anderen Gruppen, traumaexponierte Personen ohne PTBS wiederum niedrigere Werte in der Amygdala, aber höhere Werte im orbitofrontalen Kortex (OFC) im Vergleich zu den beiden anderen Gruppen [117]. In einer weiteren PET-Studie, an der in einem transdiagnostischen Ansatz nicht nur PTBSPatient*innen, sondern auch Patient*innen mit generalisierter Angststörung und Major Depression teilnahmen, war die Verfügbarkeit von $\kappa$-Opiatrezeptoren mit dem Dysphoriesymptomcluster korreliert [150].

- Die kurzfristigen adaptiven Vorteile der opioidvermittelten Stressreaktionen müssen gegen die hin- derlichen Langzeiteffekte einer prolongierten Dissoziation aufgerechnet werden. Der zustandsabhängige erhöhte Opiatgehalt während einer Traumaexposition ist möglicherweise in einen pathogenetischen Kontext zu stellen, wenn Personen mit PTBS im weiteren Verlauf eine Tendenz zeigen, nicht nur verstärkt Opiate zu konsumieren, sondern auch Situationen mit einem hohen Retraumatisierungsrisiko aufzusuchen (,trauma addiction“) [103, 104, 167]. Diese auffällige Verhaltenstendenz ist möglicherweise bei jenen PTBS-Personen mit hohem Score für „riskante Verhaltensweisen“ (Cluster D) besonders ausgeprägt [178]. In Kasuistiken wird in diesem Zusammenhang speziell über Soldaten berichtet, die schwer traumatisiert aus einem Kampfeinsatz zurückkehren und sich trotz ausgeprägter PTBS-Symptomatik erneut für ein hoch riskantes Kriegsengagement melden. Sie schildern nicht selten einen „Rausch“, den sie während eines aktiven Gefechts erleben [33, 132, 177]. Für den Verlauf von PTBS und koexistenter Opiat-Konsumstörung ist ein sich wechselseitig verstärkender neurobiologischer Ciculus vitiosus charakteristisch $[14,42,58]$.

Zusammenfassend ist für das Verständnis der Koexistenz/Komorbidität von PTBS und Substanzkonsumstörung in neurobiologischer Sicht allgemein festzuhalten, dass beide Störungen innerhalb gemeinsam neuronaler Strukturen und Mechanismen interagieren, wobei ein vermindertes Belohnungsempfinden und eine sensitivierte Stressreagibilität als klinische Merkmale hervorstechen. Spezifischen Effekten einzelner Substanzen auf die PTBS und deren Verlauf ist in dieser allgemeinen neurobiologischen Dynamik differentiell Rechnung zu tragen.

Die bisherige Diskussion der Koexistenz/Komorbidität von PTBS und Substanzkonsumstörung galt der Untersuchung in einer bidirektionalen Wirkrichtung, mit einem Blick auf Substanzkonsumstörung als Risikofaktor für Trauma und PTBS zum einen, auf Trauma und PTBS als Risikofaktor für Substanzkonsumstörung zum anderen. Sie betonte ferner auffällige Konvergenzen in den neurobiologischen Prozessen beider Störungen. Es ist aber auch ein Analysefokus möglich, der auf Faktoren gerichtet ist, die möglicherweise beiden Störungen gemeinsam zugrunde liegen. Hierbei sind vor allem genetische Aspekte, bestimmte Persönlichkeitsdimensionen und spezielle Aspekte der frühen Entwicklung näher ins Auge zu fassen.

\section{Genetische und epigenetische Faktoren als gemeinsame Basis von PTBS und Substanzkonsumstörungen}

Sowohl die PTBS als auch Substanzkonsumstörungen (allgemein, speziell) zeichnet eine vielfältige genetische Basis aus, die je für sich eine besondere Vulnerabilität versus Resilienz unter definierten Umwelt- 
einflüssen epigenetisch mitbestimmt (PTBS: [48, 81, 122, 137, 170]; Substanzkonsumstörungen allgemein: [11, 23, 64, 71, 189]; Alkohol: [43] Nikotin: [148]; Cannabis: [131]; Psychostimulantien: [89, 149]); Opiate: [24, 202]). Die genetische Forschung hat bisher zur Koexistenz/Komorbidität von PTBS und definierten Substanzkonsumstörungen erst einige wenige Studien vorgelegt:

- Für die Koexistenz von PTBS und Alkoholabhängigkeit konnte in Zwillingsstudien eine hohe genetische Überlappung nachgewiesen werden. An einem großen Sample weiblicher Zwillinge (PTBS + Alkoholmissbrauch; $N=3768$; Alter: 18-29 Jahre) erklärten additive Alkoholismus-assoziierte genetische Einflüsse über $70 \%$ der PTBS-Varianz. Die verbleibende PTBS-Varianz war auf Individuenspezifische und nicht auf gemeinsam geteilte Umweltfaktoren zurückzuführen. Die genetische Korrelation zwischen PTBS und Alkoholkonsumstörung betrug 0,54 [163]. Sie lag bei diesem Sample von Frauen höher als bei früheren Untersuchungen an männlichen Zwillingen (PTBS+Alkohol-, Cannabismissbrauch) aus dem Vietnam Era Twin Registry [101, 125, 210, 211]. Für ein männliches ZwillingsSample (Vietnam Era Twin Registry) stellte sich eine ähnliche genetische Überlappung für die Koexistenz von PTBS und Nikotinabhängigkeit dar [102].

- Mehrere molekulargenetische Studien konzentrierten sich auf den möglichen Einfluss spezieller genetischer Varianten. Die Relevanz von Genpolymorphismen für die $\alpha_{2}$-Untereinheit des GABARezeptors (GABA $\alpha_{2}$ ) wurde sowohl für die PTBS als auch für den missbräuchlichen Konsum von Alkohol, Kokain und Heroin nachgewiesen [55]. Polymorphismen des GABA-Transporter-Gens ${ }_{1}$ (GAT1), das in einer engen Verbindung mit der Enkodierung von emotionalen und Angsterinnerungen steht, wurden als signifikant sowohl für die Assoziation PTBS-Depression als auch für die Assoziation PTBS-Substanzmissbrauch erkannt [27]. Ein $\alpha$-Synuclein Polymorphismus (SNCA rs356195) vermittelte den Zusammenhang zwischen PTBSSymptomatik und riskantem Alkoholkonsum, war aber nicht mit Aggressionsmaßen eines externalisierenden Verhaltens assoziiert [69]. Ein Polymor-

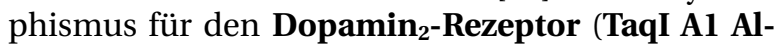
lel) konnte in seinem Einfluss auf PTBS-Patienten mit starkem Alkoholkonsum identifiziert werden [213]. Dieser Polymorphismus war auch mit einem ausgeprägten Belohnungsdefizit [22] und tierexperimentell mit dem Merkmal Impulsivität zu verbinden [41]. Der für die allgemeine Stressregulation bedeutsame 5-HTTLPR Polymorphismus des Serotonintransporters scheint bei PTBS auch mit einem verstärkten Alkoholkonsum gekoppelt $\mathrm{zu}$ sein [94]. Für die Assoziation von PTBS und Nikotinabhängigkeit wurden Genvarianten CHRNA5-
A3-B4 des nikotinergen Acetylcholin-Rezeptors diskutiert [34].

- Eine Reihe von gemeinsam (>1\%) vorkommenden Einzel-Nukleotidpolymorphismen (SNPs) üben ebenfalls moderate genetische Einflüsse sowohl auf die PTBS-Symptomatik nach einem interpersonellen Trauma als auch auf eine allgemeine Vulnerabilität für Substanzmissbrauch aus [141]. Genomweite Assoziationsstudien deckten signifikante Loci für einen klinisch breit definierten Angst-Phänotypus und eine allgemeine Vulnerabilität für missbräuchlichen Substanzkonsum auf, die auch für einzelne Untertypen (AlkoholabhängigkeitAngst: 9q33.1-q33.2, LOD =3,054; Drogenabhängigkeit-Angst: $18 \mathrm{p} 11.23-\mathrm{p} 11.22, \mathrm{LOD}=3,425)$ bestätigt werden konnten [79]. GWAS-Analysen im Rahmen des Psychiatric Genomics Consortium ermittelten mit der Methode des Kopplungsungleichgewichts (cross-trait linkage disequilibrium (LD) score regression) über je große Samples von PTBS ( $N=174.659)$ und Alkoholabhängigkeit $(N=38.686)$ signifikante Korrelationen von weiteren gemeinsam geteilten Genorten [171].

Zusammenfassend ist festzuhalten, dass die Erforschung von gemeinsam geteilten epigenetischen Einflüssen auf die Komorbidität von PTBS und Substanzkonsumstörungen derzeit in ersten Anfängen steht [20].

\section{Persönlichkeitsdimensionen als gemeinsame Basis von PTBS und Substanzkonsumstörungen}

Die empirische Forschung zeigt, dass die Persönlichkeitscharakteristika von Individuen auch bedeutsam genetisch mitdeterminiert sind. Genetische Einflüsse prägen in Interaktionen mit einschneidenden psychosozialen Lernerfahrungen insbesondere während der frühen Entwicklungsjahre grundlegende Dimensionen der Persönlichkeit [129]. Bestimmte Persönlichkeitsmerkmale und Verhaltenstendenzen sind als behaviorale Endophänotypen geeigneter, die komplexen, vielfach vermittelten Zusammenhänge von Genetik/Epigenetik und definierten psychischen Störungen für eine klinische Perspektive zu veranschaulichen [66]. Dies trifft auch auf die Komorbidität von PTBS und Substanzkonsumstörungen zu. Mehrere Persönlichkeitsdimensionen werden als Mediatorvariablen für diesen Zusammenhang diskutiert [121]:

- Impulsivität beschreibt eine Verhaltenstendenz, auf einen emotionalen oder motivationalen Reiz hin ohne sorgfältige Evaluation und Planung riskant, vorschnell und situationsinadäquat $\mathrm{zu}$ handeln. Impulsivität ist ein stark genetisch determiniertes Merkmal und ist sowohl bei Patienten mit PTBS als auch mit Substanzkonsumstörungen in besonders belasteten Subgruppen nachweisbar. Impulsivität ist mit einer niedrigen Dopamin-Baselineaktivität 
im Nucleus accumbens auf neutrale Reize, aber mit einer überschießenden striatalen dopaminergen Antwort auf prominente emotionale Reize und einer reduzierten präfrontalen Aktivierung assoziiert [41, 107]. Mit Impulsivität eng verwandte psychologische Konstrukte sind Reagibilität auf Hinweisreize („,ue reactivity“) oder emotionale Dringlichkeit („emotional urgency“). Patient*innen mit der Dualdiagnose von PTBS und Drogenkonsumstörungen (v. a. Kokain) zeichnen sich in diesen Konstrukten durch hohe Werte aus [157, 175].

- Angstsensitivität ist eng mit einer subjektiven Bedrohungswahrnehmung zu verknüpfen. Sie verweist auf eine genetisch fundierte besondere Furcht vor Symptomen einer allgemeinen physiologischen Erregung [166]. Sie geht mit kognitiven Erwartungstendenzen einher, die Unheil und Bedrohung ankündigen. Sie führt zu einer Katastrophisierungshaltung und bedingt ein ausgeprägtes Vermeidungsverhalten [12]. Angstsensitivität ist ein kognitiv-affektives Brückenkonzept, das sowohl für Substanzkonsumstörungen als auch für PTBS von empirisch nachgewiesener Relevanz ist [196].

Zusammenfassend sind beide behaviorale Endophänotypen von Impulsivität, Reagibilität auf Hinweisreize, emotionale Dringlichkeit einerseits und Angstsensitivität andererseits als Brückenkonzepte zu verstehen. Sie liegen sowohl externalisierenden als auch internalisierenden Störungen zugrunde, die in einer entwicklungspsychopathologischen Perspektive zwei polare Vorläufersyndrome von PTBS und Substanzkonsumstörung gleichermaßen bilden [209].

\section{Stressoren/Traumata in der frühen Entwicklung als gemeinsame Basis von PTBS und Substanzkonsumstörungen}

Multiple Stressoren und Traumata während der ersten Entwicklungsjahre haben sich epidemiologisch als wichtige Prädiktoren für den seelischen und körperlichen Gesundheitsstatus im Erwachsenenalter erwiesen. Hierbei begründen zahlreiche Gen-Umwelt-Interaktionen grundlegende Risikofaktoren (im Zusammenspiel mit protektiven Faktoren), die sowohl über allostatische Anpassungen der psychobiologischen Reaktionssysteme des Organismus als auch über gebahnte Verhaltensstile (Essen, Bewegung, Lifestyle, Alkohol, Drogen) zu intermediären Risikozuständen führen und schließlich in definierte psychische Störungen und somatische Krankheiten einmünden können [93]. Diese komplexen Entwicklungszusammenhänge in der Perspektive der Lebenszeitspanne sind sowohl für die PTBS als auch für Substanzkonsumstörungen empirisch gesichert nachgewiesen [115, 185, 200]. Allgemein ist für eine Koexistenz/Komorbidität von PTBS und Substanzkonsumstörung hervorzuheben:
- Effekte früher chronischer Stressoren und Traumatisierungen betreffen grundlegende Veränderungen auf allen Analyseebenen, die von der zellulären Signalgebung bis zur Verhaltensexpression reichen. Sie schließen eine Vielzahl von Neurotransmittersystemen, Mechanismen der Stressvermittlung wie HPA-Achse (dysfunktional: „Hyperkortisolismus“, „Hypokortisolismus“), Neuroinflammation (chronic low inflammation), autonomes Nervensystem (Dysbalance zwischen dominanten sympathischen und reduzierten parasympathischen Einflüssen) und zahlreiche neuronale Regelkreise des Gehirns ein. Einzelne betroffene Hirnregionen weisen je eigene Reifungs- und Entwicklungspfade auf und sind wiederum für eine Unzahl von distinkten Verhaltensformen verantwortlich mit ebenfalls eigenständigen weiteren Entwicklungspfaden. Einige Gehirnareale kodieren traumatische Informationen, die erst später zu Verhaltensauffälligkeiten führen. Bedeutsam erscheint in diesem Zusammenhang, dass definierte neuronale Strukturen, die im Erwachsenenalter eine zentrale Rolle in der Vermittlung traumatischer Erfahrungen spielen, wie insbesondere Amygdala, Hippokampus, und vor allem präfrontaler Kortex in frühen Abschnitten der Entwicklung noch höchst unreif sind. Frühe Traumatisierungen können aber die weitere Entwicklung dieser Strukturen in ihrer Funktionalität signifikant bahnen. Ihre potenziell pathogenen Auswirkungen werden erst sehr viel später als atypische Stressreaktionen erkennbar [186, 187].

- Traumata in diesen frühen Entwicklungsabschnitten betreffen in erster Linie das neuronale Bindungssystem. Dieses ist evolutionär so angelegt, dass es ein Baby inhärent dazu drängt, quasi unter allen, selbst unter traumatischen Umständen einen stabilen Kontakt zu einer Bindungsfigur aus Überlebensgründen aufrecht zu erhalten. Auch höchst widrige, schmerzhafte Erfahrungen werden in diese primäre Beziehungsform mit eingebunden. Von Geburt an steht eine reichhaltige noradrenerge Neurotransmission (Locus coeruleus) für diese basalen Lernvorgänge zur Verfügung. Die strukturelle und funktionelle Unreife jener Gehirnstrukturen, die in späteren Entwicklungsabschnitten ein Vermeidungsverhalten organisieren, wie insbesondere die Amygdala, verhindert zu diesem frühen Zeitpunkt eine effiziente behaviorale Strategie, sich von einer traumatisierenden Bindungsfigur zurückziehen zu können. Im Gegenteil, die Beziehung zu ihr ist trotz niedriger Qualität der elterlichen Fürsorge und wiederholter traumatischer Einwirkungen sogar besonders robust. Trotzdem sind jene Systeme, die in frühen Entwicklungsabschnitten noch weitgehend unreif sind, in ihrer Vorprägung von großer Tragweite in späteren Phasen der Reifung und Entwicklung [139]. Sie werden prominent zu Zeiten demaskiert, in denen weitere Stressoren oder Traumata einwirken. Mit einem überaktiven System 
der Bedrohungswahrnehmung und -bewertung (kortiko-amygdalär), einem bedeutsam reduzierten Belohnungssystem (kortiko-basalganglionär) und einem stark eingeschränkten höher-kortikalen Kontroll- und Exekutivsystem (präfrontaler Kortex) werden nicht nur Vulnerabilitäten aus der frühen traumatische Entwicklungsgeschichte hinsichtlich weiterer Stressoren und Traumata in spätere Lebensabschnitte transportiert, es werden auch die Chancen zu deren erfolgreicher Verarbeitung drastisch verringert [112].

- Die Konsequenzen von Bindungstraumata für eine besondere Vulnerabilität zu einem suchtfördernden Verhaltensstil sind in rezenten Übersichten zusammengestellt worden. Sowohl auf molekularer, neuroendokriner und behavioraler Untersuchungsebene lassen sich entscheidende Veränderungen im dopaminergen System von Belohnung und behavioraler Habitbildung, im Oxytocin-gesteuerten Bindungssystem sowie im Glukokortikoid-vermittelten Stresssystem markieren. Diese Veränderungen können sich bei unterschiedlichen Mustern einer beeinträchtigten Bindung differentiell auf prominente neurobiologische und behaviorale Aspekte auswirken, wenngleich im klinischen Kontext große Überlappungen vorkommen können [2, 98, 182]:

- Frühe Vernachlässigung geht mit einer reduzierten Expression von DA-Rezeptoren und reduzierten Dopaminproduktion einher. Hierzu ist ein stark verringertes Belohnungsempfinden im ventralen Striatum grundlegendes Korrelat. In einer Perspektive der psychopathologischen Vulnerabilität sind eine von Anhedonie geprägte Depression, ein erhöhtes „sensation seeking“ mit riskanten Verhaltensstilen sowie eine erhöhte Disposition für einen Konsum von Stimulantien (Kokain, Amphetamine, Alkohol, Nikotin) hervorzuheben.

- Unsichere Bindung ist mit einer reduzierten Expression von Oxytocin-Rezeptoren und reduzierten Oxytocinproduktion assoziiert. Eine Sensibilisierung gegenüber sozialen und interpersonalen Reizen mit veränderten neuronalen Aktivitäten im präfrontalen Kortex und Hypothalamus ist kennzeichnend. Psychopathologisch sind eine verstärkte Neigung zu sozialer Isolation, ein gestörtes Bindungsverhalten, ein prominenter emotionaler Schmerz sowie eine Disposition für einen Konsum von Opiaten charakteristisch.

- Früher Missbrauch ist mit einer reduzierten Expression von Glukokortikoid-Rezeptoren und erhöhten CRF-Aktivität korreliert. Veränderungen in den Stresssystemen betreffen vor allem die Aktivität der Amygdala und der HPA-Achse. Psychopathologisch imponieren verstärkte Angst- und PTBS-Symptome, Zeichen eines ungelösten Traumas (komplexe PTBS) sowie eine Disposition für einen Konsum von tranquilisierenden Substanzen (Alkohol, Cannabis, Benzodiazepine, etc.).
Zusammenfassend tragen all diese akzentuierten neurobiologischen und behavioralen Profile als Resultanten eines früh gestörten Bindungsverhaltens $\mathrm{zu}$ einem allgemein und differentiell erhöhten Suchtrisiko bei. Diese früh erworbenen neurobiologischen und behavioralen Dispositionen kommen auch während der Jugend und im Erwachsenenalter im Kontext eines unsicheren Bindungsverhaltens zum Tragen. Sie können unter ungünstigen psychosozialen Entwicklungsbedingungen oder belastenden Lebenssituationen weiter verschärft werden und so $\mathrm{zu}$ einem Circulus vitiosus von Trauma-Sucht-Trauma-Sucht führen.

\section{Schlussbemerkungen}

Trauma, PTBS und Sucht definieren komplexe Herausforderungen in der Versorgung. Ihre häufige Koexistenz führt zu schwerwiegenderen akuten klinischen Symptombildern mit zahlreichen, oft notfallmäßigen Hospitalisierungen. Sie trägt zu dramatisch ungünstigeren Verläufen auf allen biopsychosozialen Ebenen bei. Ein differenziertes neurobiologisches, psychologisches und soziales Verständnis der Komorbidität von PTBS und Substanzkonsumstörungen ist entscheidende Voraussetzung für eine vorteilhaftere Behandlungspraxis. Diese ist derzeit mehrheitlich noch durch getrennte Behandlungsansätze für PTBS zum einen, für Substanzkonsumstörung zum anderen mit je niedrigen therapeutischen Ansprechraten und hohen Abbruchquoten gekennzeichnet. Eine systematische und simultane Beachtung der je besonderen Ansprüche aus beiden Störungen ist in einem spezialisierten Therapiesetting $\mathrm{zu}$ fordern. Ein solcher integrativer Behandlungsansatz wird sich sowohl auf empirisch erprobte Trauma-psychotherapeutische Verfahren stützen und gleichzeitig innovative pharmakologische Strategien zu Therapie, Substitution und Prophylaxe der koexistenten Sucht einsetzen [6, 8, 174, 195].

Funding Open access funding provided by Medical University of Graz.

Interessenkonflikt H.-P. Kapfhammer gibt an, dass kein Interessenkonflikt besteht.

Open Access Dieser Artikel wird unter der Creative Commons Namensnennung 4.0 International Lizenz veröffentlicht, welche die Nutzung, Vervielfältigung, Bearbeitung, Verbreitung und Wiedergabe in jeglichem Medium und Format erlaubt, sofern Sie den/die ursprünglichen Autor(en) und die Quelle ordnungsgemäß nennen, einen Link zur Creative Commons Lizenz beifügen und angeben, ob Änderungen vorgenommen wurden.

Die in diesem Artikel enthaltenen Bilder und sonstiges Drittmaterial unterliegen ebenfalls der genannten Creative Commons Lizenz, sofern sich aus der Abbildungslegende nichts anderes ergibt. Sofern das betreffende Material nicht unter der genannten Creative Commons Lizenz steht und die betreffende Handlung nicht nach gesetzlichen Vorschriften erlaubt ist, ist für die oben aufgeführten Weiterverwendungen 
des Materials die Einwilligung des jeweiligen Rechteinhabers einzuholen.

Weitere Details zur Lizenz entnehmen Sie bitte der Lizenzinformation auf http://creativecommons.org/licenses/by/4. 0/deed.de.

\section{Literatur}

1. Alexander AC, Ward KD. Understanding postdisaster substance use and psychological distress using concepts from the self-medication hypothesis and social cognitive theory. JPsychoactive Drugs. 2018;50:177-86.

2. Alvarez-Monjaras M, Mayes LC, Potenza MN, Rutherford HJ. A developmental model of addictions: integrating neurobiological and psychodynamic theories through the lens of attachment. Attach Hum Dev. 2019;21:616-63.

3. Andrews NCZ, Motz M, Bondi BC, et al. Using a developmental-relational approach to understand the impact of interpersonal violence in women who struggle with substance use. Int J Environ Res Public Health. 2019;16:4861. https:// doi.org/10.3390/ijerph16234861.

4. AshokAH, Mizuno Y, Volkow ND, Howes OD. Association of stimulant use with dopaminergic alterations in users of cocaine, amphetamine, or methamphetamine: a systematic review and meta-analysis. JAMA Psychiatry. 2017;74:511-9.

5. Averill LA, Purohit P, Averill CL, et al. Glutamate dysregulation and glutamatergic therapeutics for PTSD: evidence from human studies. Neurosci Lett. 2017;649:147-55.

6. BackSE, Killeen T, Badour CL, Flanagan JC, et al. Concurrent treatment of substance use disorders and PTSD using prolonged exposure: a randomized clinical trial in military veterans. Addict Behav. 2019;90:369-77.

7. Bahji A, Forsyth A, Groll D, Hawken ER. Efficacy of 3,4-methylenedioxymethamphetamine (MDMA)-assisted psychotherapyforposttraumaticstress disorder:asystematic review and meta-analysis. Prog Neuropsychopharmacol Biol Psychiatry. 2020;96:109735. https://doi.org/10.1016/j. pnpbp.2019.109735.

8. Bailey K, Trevillion K, Gilchrist G. What works for whom and why: a narrative systematic review of interventions for reducing post-traumatic stress disorder and problematic substance use among women with experiences of interpersonal violence. J Subst Abuse Treat. 2019;99:88-10.

9. BasedowLA, Kuitunen-PaulS, RoessnerV, GolubY.Traumatic events and substance use disorders in adolescents. Front Behav Neurosci.2020;14:6. https://doi.org/10.3389/fnbeh. 2020.00006.

10. Batra A, Düerkop M, Gertzen M, et al. Nikotin. In: Soyka M, Batra A, Heinz A, Moggi F, Walter M, Hrsg. Suchtmedizin. München:Elsevier/Urban \&Fischer; 2020. S. 237-58.

11. Beayno A, El Hayek S, Noufi P, et al. The role of epigenetics in addiction: clinical overviewand recentupdates. Methods MolBiol. 2019;2011:609-31.

12. Berman NC, Wheaton MG, McGrath P, Abramowitz JS. Predicting anxiety: the role of experiential avoidance and anxiety sensitivity. J Anxiety Disord. 2010;24:109-13.

13. Bernardy NC, Friedman MJ. Pharmacological management of posttraumatic stress disorder. Curr Opin Psychol. 2017;14:116-21.

14. Bernardy NC, Montaño M. Opioid use among individuals with posttraumatic stress disorder. PTSD Res Q. 2019;30(1):1-8.

15. Bernardy NC, Lund BC, Alexander B, et al. Gender differences in prescribing among veterans diagnosed with posttraumatic stress disorder. J Gen Intern Med. 2013;28(Suppl 2):542-8.
16. Berridge KC. Evolving concepts of emotion and motivation. Front Psychol. 2018;9:1647.https://doi.org/10.3389/fpsyg. 2018.01647.

17. Berridge KC, Robinson TE. Liking, wanting and the incentive-sensitization theory of addiction. Am Psychol. 2016;71:670-9.

18. Bilevicius E, Sommer JL, Asmundson GJG, El-Gabalawy R. Posttraumatic stress disorder and chronic pain are associated with opioid use disorder: results from a 2012-2013 American nationally representative survey. Drug Alcohol Depend.2018;188:119-25.

19. Black N, Stockings E, Campbell G, et al. Cannabinoids for the treatment of mental disorders and symptoms of mental disorders: a systematic review and meta-analysis. Lancet Psychiatry. 2019;6:995-1010.

20. Blacker CJ, Frye MA, Morava E, et al. A review of epigenetics of PTSD in comorbid psychiatric conditions. Genes (Basel). 2019;10:140. https://doi.org/10.3390/genes10020140.

21. Blanco C, Xu Y, Brady K, et al. Comorbidity of posttraumatic stress disorder with alcohol dependence among US adults: results from National Epidemiological Survey on Alcohol and Related Conditions. Drug Alcohol Depend. 2013;132:630-8.

22. Blum K, Gondré-Lewis MC, Modestino EJ, et al. Understanding the scientific basis of post-traumatic stress disorder (PTSD): precision behavioral management overrides stigmatization. Mol Neurobiol. 2019; https://doi.org/10. 1007/s12035-019-1600-8.

23. Blum K, Baron D, Lott L, Ponce JV, et al. In search of reward deficiency syndrome (RDS)-free controls: the "Holy Grail" in genetic addiction risk testing. Curr Psychopharmacol. 2020;9:7-21.

24. Bodnar RJ. Endogenous opiates and behavior: 2018. Peptides. 2020;132:170348. https://doi.org/10.1016/j.peptides. 2020.170348.

25. Borsook D, Youssef AM, Simons L, et al. When pain gets stuck: the evolution of pain chronification and treatment resistance. Pain. 2018;159:2421-36.

26. Boscarino JA, Kirchner HL, Hoffman SN, Sartorius J, Adams RE. PTSD and alcohol use after the World Trade Center attacks: a longitudinal study. J Trauma Stress. 2011;24:515-25.

27. Bountress KE, Wei W, Sheerin C, et al. Relationships between GAT1 and PTSD, depression, and substance use disorder. Brain Sci. 2017;7:6. https://doi.org/10.3390/ brainsci7010006.

28. Bountress KE, Cusack SE, Sheerin CM, et al. Alcohol consumption, interpersonal trauma, and drinking to cope with trauma-related distress: an auto-regressive, cross-lagged model. PsycholAddict Behav. 2019;33:221-31.

29. Bremner JD, SouthwickSM, DarnellA, CharneyDS. Chronic PTSD in Vietnam combat veterans: course of illness and substance abuse. Am J Psychiatry. 1996;153:369-75.

30. Breslau N. Epidemiologic studies of trauma, posttraumatic stress disorder, and other psychiatric disorders. Can J Psychiatry. 2002;47:923-9.

31. Breslau N, Davis GC, Schultz LR. Posttraumatic stress disorder and the incidence of nicotine, alcohol, and other drug disorders in persons who have experienced trauma. Arch Gen Psychiatry. 2003;60:289-94.

32. Brunault P, Lebigre K, IdbrikF, et al. Posttraumatic stress disorder is a risk factor for multiple addictions in police officers hospitalized for alcohol. EurAddict Res. 2019;25:198-206.

33. Campbell MS, Ryan M, Wright D, et al. Postdeployment PTSDandaddictivecombatattachmentbehaviorsinUSmilitary service members. Am J Psychiatry. 2016;173:1171-6. 
34. Chen LS, Xian H, Grucza RA, et al. Nicotine dependence and comorbid psychiatric disorders: examination of specific genetic variants in the CHRNA5-A3-B4 nicotinic receptor genes. Drug Alcohol Depend. 2012;123(Suppl 1):S42-S51.

35. Cheng HG, Shidhaye R, Charlson F, et al. Social correlates of mental, neurological, and substance use disorders in China and India: a review. Lancet Psychiatry. 2016;3:882-99.

36. Clark CB, Reiland S, Thorne C, Cropsey KL. Relationship of trauma exposure and substance abuse to self-reported violence among men and women in substance abuse treatment. J Interpers Violence. 2014;29:1514-30.

37. Clarke DE, Gonzalez M, Pereira A, et al. The impact of knowledge on attitudes of emergency department staff towards patients with substance related presentations: a quantitative systematic review protocol. JBI Database System Rev Implement Rep. 2015;13:133-45.

38. Clausen AN, Francisco AJ, Thelen J, et al. PTSD and cognitive symptoms relate to inhibition-related prefrontal activation and functional connectivity. Depress Anxiety. 2017;34:427-36.

39. Cottler LB, Compton WM III, Mager D, et al. Posttraumatic stress disorder among substance users from the general population. Am J Psychiatry. 1992;149:664-70.

40. Dahlby L, Kerr T. PTSD and opioid use: implications for intervention and policy. Subst Abuse Treat Prev Policy. 2020;15:22.https:// doi.org/10.1186/s13011-020-00264-8.

41. Dalley JW, Robbins TW. Fractionating impulsivity: neuropsychiatricimplications. NatRevNeurosci.2017;18:158-71.

42. Danovitch I. Post-traumatic stress disorder and opioid use disorder: a narrative review of conceptual models. J Addict Dis. 2016;35:169-79.

43. DeakJD, MillerAP, Gizer IR. Genetics of alcoholuse disorder: a review. Curr Opin Psychol. 2019;27:56-61.

44. Debell F, Fear NT, Head M, et al. A systematic review of the comorbidity between PTSD and alcohol misuse. Soc Psychiatry Psychiatr Epidemiol.2014;49:1401-25.

45. Dela Peña I, Gevorkiana R, Shi WX. Psychostimulants affect dopamine transmission through both dopamine transporter-dependent and independent mechanisms. Eur J Pharmacol. 2015;764:562-70.

46. DiMaggio C, Galea S, Li G. Substance use and misuse in the aftermath of terrorism. A Bayesian meta-analysis. Addiction. 2009;104:894-904

47. Driessen M, Schulte S, Lüdecke C, et al. Trauma and PTSD in patients with alcohol, drug, or dual dependence: a multicenter study. AlcoholClin Exp Res. 2008;32:481-8.

48. Duncan LE, Cooper BN, Shen H. Robust findings from 25 years of PTSD genetics research. Curr Psychiatry Rep. 2018;20:115. https://doi.org/10.1007/s11920-018-0980-1.

49. Duval A, Lanning BA, Patterson MS. A systematic review of dating violence risk factors among undergraduate college students. Trauma ViolenceAbuse. 2020;21:567-85.

50. Dworkin ER, Bergman HE, Walton TO, et al. Co-occurring post-traumatic stress disorder and alcohol use disorder in U.S. military and veteran populations. Alcohol Res. 2018a;39:161-9.

51. Dworkin ER, Wanklyn S, Stasiewicz PR, Coffey SF. PTSD symptom presentation among people with alcohol and drug use disorders: comparisons by substance of abuse. Addict Behav. 2018b;76:188-94.

52. Elman I, Borsook D. Threat response system: parallel brain processesin painvis-à-visfearandanxiety. FrontPsychiatry. 2018;9:29. https://doi.org/10.3389/fpsyt.2018.00029.

53. ElmanI, BorsookD. Thefailing cascade:comorbid posttraumatic stress- and opioid use disorders. Neurosci Biobehav Rev.2019;103:374-83.
54. Enman NM,ZhangY, UnterwaldEM.Connecting thepathology of posttraumatic stress and substance use disorders: monoamines and neuropeptides. Pharmacol Biochem Behav. 2014;117:61-9.

55. Enoch MA, Hodgkinson CA, Yuan Q, et al. The influence of GABRA2, childhood trauma, and their interaction on alcohol, heroin, and cocaine dependence. Biol Psychiatry. 2010;67:20-7.

56. Erskine N, Daley V, Stevenson S, Rhodes B, Beckert L. Smoking prevalence increases following Canterbury earthquakes. Sci World J. 2013; https://doi.org/10.1155/2013/ 596957.

57. Ertl V, Saile R, Neuner F, Catani C. Drinking to ease the burden: a cross-sectional study on trauma, alcohol abuse and psychopathology in a post-conflict context. BMC Psychiatry. 2016;16:202. https://doi.org/10.1186/s12888016-0905-7.

58. Fareed A, Eilender P, al Haberet M. Comorbid posttraumatic stress disorder and opiate addiction: a literature review. JAddict Dis. 2013;32:168-79.

59. FeducciaAA, JeromeL, Yazar-KlosinskiB, etal.Breakthrough for trauma treatment: safety and efficacy ofMDMA-assisted psychotherapy compared to paroxetine and sertraline. Front Psychiatry. 2019;10:650. https://doi.org/10.3389/ fpsyt.2019.00650.

60. Fegert J, Freyberger HJ. Posttraumatische Belastungsstörungen (PTBS) und Traumafolgestörungen in der Adoleszenz. Fortschr Neurol Psychiatr. 2019;87:638-41.

61. Fetzner MG, McMillan KA, Sareen J, Asmundson GJG. What is the association between traumatic life events and alcohol abuse/dependence in people with and without PTSD? Findings from a nationally representative sample. Depress Anxiety. 2011;28:632-8.

62. Forbes MK, Flanagan JC, Barrett EL, et al. Smoking, posttraumatic stress disorder, and alcohol use disorders in a nationally representative sample of Australian men and women. DrugAlcohol Depend.2015;156:176-83.

63. Forehand JA, Peltzman T, Westgate CL, et al. Causes of excess mortality in veterans treated for posttraumatic stress disorder.Am J Prev Med.2019;57:145-52.

64. Fulton SL, Maze I. Translational molecular approaches in substance abuse research. Handb Exp Pharmacol. 2020;258:31-60.

65. Gelpin E, Bonne O, Peri T, et al. Treatment of recent trauma survivors with benzodiazepines: a prospective study. J Clin Psychiatry. 1996;57:390-4.

66. Gottesman II, Gould TD. The endophenotype concept in psychiatry: etymology and strategic intentions. Am J Psychiatry. 2003;160:636-45.

67. Gouzoulis-Mayfrank E, Majić T, Schaub M. Stimulanzien vomAmphetamintyp. In: Soyka M, BatraA, HeinzA, MoggiF, Walter M, Hrsg. Suchtmedizin. München: Elsevier/Urban \& Fischer;2020. S. 203-21.

68. Grant BF, Saha TD, Ruan WJ, et al. Epidemiology of DSM-5 drug use disorder: results from the National Epidemiologic Survey on Alcohol and Related Conditions-III. JAMA Psychiatry. 2016;73:39-47.

69. Guillot CR, Fanning JR, Liang T, et al. An $\alpha$-synuclein gene (SNCA) polymorphism moderates the association of PTSD symptomatology with hazardous alcohol use, but not with aggression-related measures. J Anxiety Disord. 2015;30:41-7.

70. Haller M, Chassin L. Risk pathways among traumatic stress, posttraumatic stress disorder symptoms, and alcohol and drug problems: a test of four hypotheses. Psychol Addict Behav. 2014;28:841-51. 
71. Hamilton PJ, Nestler EJ. Epigenetics and addiction. Curr Opin Neurobiol.2019;59:128-36.

72. Hämmig R, Preuss UW, Soyka M. Cannabis. In: Soyka M, Batra A, Heinz A, Moggi F, Walter M, Hrsg. Suchtmedizin. München: Elsevier, Urban \& Fischer; 2020. S. 143-75.

73. Hassan AN, Le Foll B, Imtiaz S, Rehm J. The effect of post-traumatic stress disorder on the risk of developing prescription opioid use disorder: Results from the National Epidemiologic Survey on Alcohol and Related Conditions III. Drug AlcoholDepend. 2017;179:260-6.

74. Head M, Goodwin L, Debell F, et al. Post-traumatic stress disorder and alcohol misuse: comorbidity in UK military personnel. Soc Psychiatry Psychiatr Epidemiol. 2016;51:1171-80.

75. Heerde JA, Hemphill SA. Sexual risk behaviors, sexual offenses, and sexual victimization among homeless youth: a systematic review of associations with substance use. Trauma ViolenceAbuse. 2016;17:468-89.

76. Heinz A, Batra A, Scherbaum N, Gouzoulis-Mayfrank E. Neurobiologie der Abhängigkeit. Stuttgart: Kohlhammer; 2012.

77. Hill MN, Campolongo P, Yehuda R, PatelS. Integrating endocannabinoid signaling and cannabinoids into the biology and treatment of posttraumatic stress disorder. Neuropsychopharmacology. 2018;43:80-102.

78. Hirst A, Miller-Archie SA, Welch AE, et al. Post-9/11 drugand alcohol-related hospitalizations among World Trade Center Health Registry enrollees, 2003-2010. Drug Alcohol Depend. 2018;187:55-60.

79. Hodgson K, Almasy L, Knowles EEM, et al. Genome-wide significant loci for addiction and anxiety. Eur Psychiatry. 2016;36:47-54.

80. Homish GG, Hoopsick RA, Heavey SC, et al. Drug use and hazardous drinking are associated with PTSD symptoms and symptom clusters in US Army Reserve/National Guard soldiers. Am J Addict. 2019;28:22-8.

81. Howie H, Rijal CM, Ressler KJ. A review of epigenetic contributions to post-traumatic stress disorder. Dialogues Clin Neurosci. 2019;21:417-28.

82. Hughes K, Bellis MA, Hardcastle KA, et al. The effect of multiple adverse childhood experiences on health: a systematic review and meta-analysis. Lancet Public Health. 2017;2:e356-e66.

83. Jaffe AE, Blayney JA, Bedard-Gilligan M, Kaysen D. Are trauma memories state-dependent? Intrusive memories following alcohol-involved sexual assault. Eur J Psychotraumatol. 2019;10:1634939. https://doi.org/10.1080/ 20008198.2019.1634939.

84. Jerome L, Feduccia AA, Wang JB, et al. Long-term follow-up outcomes of MDMA-assisted psychotherapy for treatment of PTSD: a longitudinal pooled analysis of six phase 2 trials. Psychopharmacology (Berl). 2020;237:2485-97.

85. Jetly R, Heber A, Fraser G, Boisvert D. The efficacy of nabilone, a synthetic cannabinoid, in the treatment of PTSD-associated nightmares: a preliminary randomized, double-blind, placebo-controlled cross-over design study. Psychoneuroendocrinology. 2015;51:585-8.

86. Johnson SD. Substance use, post-traumatic stress disorder and violence. Curr Opin Psychiatry. 2008;21:242-6.

87. Johnson SD, Striley C, Cottler LB. The association of substance use disorders with trauma exposure and PTSD among African American drug users. Addict Behav. 2006;31:2063-73.

88. JoudreyPJ, Khan MR, WangEA, etal.A conceptual model for understanding post-release opioid-related overdose risk. Addict Sci Clin Pract. 2019;14:17. https://doi.org/10.1186/ s13722-019-0145-5.
89. Kalda A, Zharkovsky A. Epigenetic mechanisms of psychostimulant-induced addiction. Int Rev Neurobiol. 2015;120:85-105.

90. Kalivas PW. The glutamate homeostasis hypothesis of addiction. Nat Rev Neurosci. 2009;10(8):561-72.

91. Kalivas PW, VolkowND. Newmedications for drugaddiction hiding in glutamatergic neuroplasticity. Mol Psychiatry. 2011;16:974-86.

92. Kapfhammer HP. Akute und posttraumatische Belastungsstörung. In: Möller HJ, Laux G, Kapfhammer HP, Hrsg. Psychiatrie, Psychosomatik und Psychotherapie. 5. Aufl. Bd.3. Berlin, Heidelberg: Springer; 2017. S. 1965-2040.

93. Kapfhammer HP. Depression, Angst, traumatischer Stress und somatische Morbidität. 2021.in Vorbereitung.

94. Kaufman J, Yang BZ, Douglas-Palumberi H, et al. Genetic and environmental predictors of early alcohol use. Biol Psychiatry. 2007;61:1228-34.

95. Kearns NT, Carl E, Stein AT, et al. Posttraumatic stress disorder and cigarette smoking: a systematic review. Depress Anxiety. 2018;35:1056-72.

96. Kessler RC, Sonnega A, Bromet E, et al. Posttraumatic stress disorder in the National Comorbidity Survey. Arch Gen Psychiatry. 1995;52:1048-60.

97. Khantzian EJ. Addiction as a self-regulation disorder and the role of self-medication. Addiction. 2013;108:668-9.

98. Kim S, KwokS, Mayes LC, et al. Early adverse experience and substance addiction: dopamine, oxytocine, and glucocorticoid oathways. Ann NYAcad Sci. 2017;1394:74-91.

99. Kline NK, Berke DS, Rhodes CA. Self-blame and PTSD following sexual assault: a longitudinal analysis. J Interpers Violence.2018; https://doi.org/10.1177/0886260518770652.

100. Kobayashi TM, Patel M, Lotito M. Pharmacotherapy for posttraumatic stress disorder at a Veterans Affairs facility. AmJ Health Syst Pharm. 2015;72(11 Suppl 1):S11-S5.

101. Koenen KC, Lyons MJ, Goldberg J, et al. Co-twin control study of relationships among combat exposure, combatrelated PTSD, and other mental disorders. J Trauma Stress. 2003;16:433-8.

102. Koenen KC, Hitsman B, Lyons MJ, et al. A twin registry study of the relationship between posttraumatic stress disorder and nicotine dependence in men. Arch Gen Psychiatry. 2005;62:1258-65.

103. van der Kolk B, Greenberg M, Boyd H, Krystal J. Inescapable shock, neurotransmitters, and addiction to trauma: toward a psychobiology of post traumatic stress disorder. Biol Psychiatry. 1985;30:314-25.

104. van der KolkBA, Greenberg MS, Orr SP, Pitman RK. Endogeneous opiods, stress induced analgesia, and posttraumatic stress disorder. Psychopharmacol Bull. 1989;25:417-21.

105. Koob GF. The dark side of emotion: the addiction perspective. Eur J Pharmacol. 2015;753:73-87.

106. Koob GF, Volkow ND. Neurobiology of addiction: a neurocircuitry analysis. Lancet Psychiatry. 2016;3:760-73.

107. Kozak K, Lucatch AM, Lowe DJE, et al. The neurobiology of impulsivity and substance use disorders: implications for treatment. Ann NYAcad Sci. 2019;1451:71-9.

108. Krediet E, Bostoen T, Breeksema J, et al. Reviewing the potential of psychedelics for the treatment of PTSD. Int J Neuropsychopharmacol. 2020; https://doi.org/10.1093/ ijnp/pyaa018.

109. Kulka RA, Schlenger WE, FairbankJA, et al. Trauma and the Vietnam War generation: report of findings from the National Vietnam Veterans Readjustment Study. Philadelphia: Brunner/Mazel; 1990.

110. Kuntsche E, Kuntsche S, Thrul J, Gmel G. Binge drinking: health impact, prevalence, correlates and interventions. Psychol Health. 2017;32:976-1017. 
111. Kwako LE, Koob GF. Neuroclinical framework for the role of stress in addiction. Chronic Stress (Thousand Oaks). 2017; https://doi.org/10.1177/2470547017698140.

112. Lahousen T, Unterrainer HF, Kapfhammer HP. Psychobiology of attachment and trauma-some general remarks from a clinical perspective. Front Psychiatry. 2019;10:914. https://doi.org/10.3389/fpsyt.2019.00914.

113. Lee JY, Brook JS, Finch SJ, et al. Single and dual diagnoses of major depressive disorder and posttraumatic stress disorder predicted by triple comorbid trajectories of tobacco, alcohol, and marijuana use among urban adults. Subst Abus. 2019;40:221-8.

114. Lee JY, Kim SW, Kim JM. The impact of community disaster trauma: a focus on emerging research of PTSD and other mental health outcomes. Chonnam Med J. 2020a;56:99-107.

115. Lee RS, Oswald LM, Wand GS. Early life stress as a predictor of co-occurring alcohol use disorder and post-traumatic stress disorder. Alcohol Res. 2018;39:147-59.

116. Lee WK, Hayashi K, DeBeck K, et al. Association between posttraumatic stress disorder and nonfatal drug overdose. Psychol Trauma. 2020b;12:373-80.

117. Liberzon I, Taylor SF, Phan KL, et al. Altered central $\mu$-opioid receptor binding after psychological trauma. Biol Psychiatry. 2007;61:1030-8.

118. Mahan AL, Ressler KJ. Fear conditioning, synaptic plasticity and the amygdala: implications for posttraumatic stress disorder. Trends Neurosci. 2012;35:24-5.

119. Mahoney CT, Cole HE, Gilbar O, Taft CT. The role of impulsivity in the association between posttraumatic stress disorder symptom severity and substance use in male military veterans. JTrauma Stress. 2020;33:296-306.

120. Marmot $M$. The health gap: the challenge of an unequal world. London, NewYork: Bloomsbury; 2016.

121. María-Ríos CE, Morrow JD. Mechanisms of shared vulnerability to post-traumatic stress disorder and substance use disorders. Front Behav Neurosci.2020;14:6. https://doi. org/10.3389/fnbeh.2020.00006.

122. Maul S, Giegling I, Fabbri C, et al. Genetics of resilience: implications from genome-wide association studies and candidate genes of the stress response system in posttraumatic stress disorder and depression. Am J Med Genet B Neuropsychiatr Genet. 2020;183:77-94.

123. Mayer FP, Luf A, Nagy C, et al. Application of a combined approach to identify new psychoactive street drugs and decipher their mechanisms at monoamine transporters. Curr Top Behav Neurosci. 2017;32:333-50.

124. McDevitt-Murphy ME, Fields JA, Monahan CJ, Bracken KL. Drinking motives among heavy-drinking veterans with and without posttraumatic stress disorder. Addict Res Theory. 2015;23:148-55.

125. McLeod DS, Koenen KC, Meyer JM, et al. Genetic and environmental influences on the relationship among combat exposure, posttraumatic stress disorder symptoms, and alcohol use. J Trauma Stress. 2001;14:259-75.

126. Mellman TA, Bustamante V, David D, et al. Hypnotic medication in the aftermath of trauma. J Clin Psychiatry. 2002a;63:1183-4.

127. Meyerhoff DJ, Mon A, Metzler T, Neylan TC. Cortical $\gamma$-aminobutyric acid and glutamate in posttraumatic stress disorder and their relationships to self-reported sleep quality. Sleep. 2014;37:893-900.

128. Mills KL, Lynskey M, Teesson M, et al. Post-traumatic stress disorder among people with heroin dependence in the Australian treatment outcome study (ATOS): prevalence and correlates. Drug AlcoholDepend. 2005;77:243-249.
129. Montag C, Ebstein RP, Jawinski P, Markett S. Molecular genetics in psychology and personality neuroscience: on candidate genes, genome wide scans, and new research strategies. Neurosci Biobehav Rev. 2020; https://doi.org/ 10.1016/j.neubiorev.2020.06.020.

130. Müller M, Vandeleur C, Rodgers S, et al. Childhood adversities as specific contributors to the co-occurrence of posttraumatic stress and alcohol use disorders. Psychiatry Res. 2015;228:251-6.

131. Mulligan MK. Genetic factors in cannabinoid use and dependence. Adv Exp Med Biol. 2019;1162:129-50.

132. Nadelson T. Attachment to killing. J Am Acad Psychoanal. 1992;20:130-41.

133. National Academies of Sciences, Engineering and Medicine. The health effects of cannabis and cannabinoids: the current state of evidence and recommendations for research. Washington, DC: National Acacdemies Press; 2017.

134. Nawijn L, van Zuiden M, Frijling JL, et al. Reward functioning in PTSD: a systematic review exploring the mechanisms underlying anhedonia. Neurosci Biobehav Rev. 2015;51:189-204.

135. NestlerEJ.Cellularbasis ofmemoryfor addiction.Dialogues Clin Neurosci. 2013;15:431-43.

136. Neumeister A, Seidel J, Ragen BJ, Pietrzak RH. Translational evidence for a role of endocannabinoids in the etiology and treatment of posttraumatic stress disorder. Psychoneuroendocrinology. 2015;51:577-84.

137. NisarS, BhatAA, Hashem S, etal.Genetic and neuroimaging approaches to understanding post-traumatic stress disorder. Int J Mol Sci. 2020;21:4503. https://doi.org/10.3390/ ijms21124503.

138. Norman SB, Haller M, Hamblen JL, et al. The burden of cooccurring alcoholuse disorder and PTSD in U.S. militaryveterans: comorbidities, functioning, and suicidality. Psychol Addict Behav. 2018;32:224-9.

139. Opendak M, Sullivan RM. Unique infant neurobiology produces distinct trauma processing. Dev Cogn Neurosci. 2019;36:100637.https://doi.org/10.1016/j.dcn.2019. 100637.

140. Orsolini L, Chiappini S, Volpe U, et al. Use of medicinal cannabis and synthetic cannabinoids in post-traumatic stress disorder (PTSD): a systematic review. Medicina (Kaunas). 2019;55:525. https://doi.org/10.3390/medicina55090525.

141. Palmer RH, Nugent NR, Brick LA, et al. Evidence of shared genome-wide additive genetic effects on interpersonal trauma exposure and generalized vulnerability to drug dependence in a population of substance users. J Trauma Stress. 2016;29:197-204.

142. Papini S, Sullivan GM, Hien DA, et al. Toward a translational approach to targeting the endocannabinoid system in posttraumatic stress disorder: a critical review of preclinical research. Biol Psychol. 2015;104:8-18.

143. Patel RS, Elmaadawi A, Nasr S, Haskin J. Comorbid posttraumatic stress disorder and opioid dependence. Cureus. 2017;9:e1647.https://doi.org/10.7759/cureus.1647.

144. Peltonen K, Ellonen N, Pitkänen J, et al. Trauma and violent offending among adolescents: a birth cohort study. Epidemiol Community Health. 2020; https://doi.org/10.1136/ jech-2020-214188.

145. PenningtonDL,AbéC,BatkiSL, MeyerhoffDJ.Apreliminary examination of cortical neurotransmitter levels associated with heavy drinkingin posttraumatic stress disorder. PsychiatryRes. 2014;224:281-7.

146. Pericot-Valverde I, Elliott RJ, Miller ME, et al. Posttraumatic stress disorder and tobacco use: a systematic review and meta-analysis. Addict Behav. 2018;84:238-47. 
147. Peter-Hagene LC, Ullman SE. Longitudinal effects of sexual assault victims' drinking and self-blame on posttraumatic stress disorder. J Interpers Violence. 2016; https:/ / doi.org/ $10.1177 / 0886260516636394$.

148. Picciotto MR, Kenny PJ. Mechanisms of nicotine addiction. Cold Spring Harb Perspect Med. 2020; https://doi.org/10. 1101/cshperspect.a039610.

149. Pierce RC, Fant B, Swinford-Jackson SE, et al. Environmental, genetic and epigenetic contributions to cocaine addiction. Neuropsychopharmacology. 2018;43:1471-80.

150. Pietrzak RH, Naganawa M, Huang Y, et al. Association of in vivo k-opioid receptor availability and the transdiagnostic dimensional expression of trauma-related psychopathology. JAMAPsychiatry. 2014;71:1262-70.

151. Pietrzak RH, Goldstein RB, Southwick SM, Grant BF. Prevalence and Axis I comorbidity of full and partial posttraumatic stress disorder in the United States: results from Wave 2 of the National Epidemiologic Survey on Alcohol and Related Conditions. JAnxiety Disord. 2011;25:456-65.

152. Pollice R, Bianchini V, Roncone R, Casacchia M. Marked increase in substance use among young people after L'Aquila earthquake. Eur Child Adolesc Psychiatry. 2011;20:429-30.

153. Potvin S, Pelletier J, Grot S, et al. Cognitive deficits in individuals with methamphetamine use disorder: a metaanalysis. Addict Behav. 2018;80:154-60.

154. de Quervain DF, Roozendaal B, Nitsch RM, et al. Acute cortisone administration impairs retrieval of long-term spatial memory. Nat Neurosci. 2000;3:313-4.

155. RalevskiE, SouthwickS, Jackson E, etal. Trauma- and stressinduced response in veterans with alcohol dependence and comorbid post-traumatic stress disorder. Alcohol Clin Exp Res. 2016;40:1752-60.

156. Rasmussen IS, Arefjord K, Winje D, Dovran A. Childhood maltreatment trauma: a comparison between patients in treatment for substance use disorders and patients in mental health treatment. Eur J Psychotraumatol. 2018;9:1492835. https://doi.org/10.1080/20008198.2018. 1492835.

157. Read JP, Bachrach RL, Wardell JD, Coffey SF. Examining cognitive processes and drinking urge in PTSD. Behav Res Ther. 2017;90:159-68.

158. Reed PL, AnthonyJC, Breslau N. Incidence of drug problems in young adults exposed to trauma and posttraumatic stress disorder: Do early life experiences and predispositions matter? Arch Gen Psychiatry. 2007;64:1435-42.

159. Reiff CM, Richman EE, Nemeroff CB, et al. Psychedelics and psychedelic-assisted psychotherapy. Am J Psychiatry. 2020;177:391-410.

160. Rodriguez L, Read JP. Momentary emotional responding and emotion regulation in PTSD-related drinking urge. Exp Clin Psychopharmacol.2020;28:99-111.

161. Roozendaal B, McEwen BS, Chattarji S. Stress, memory and the amygdale. Nat Rev Neurosci. 2009;10:423-33.

162. Rosso IM, Weiner MR, Crowley DJ, et al. Insula and anterior cingulate GABA levels in posttraumatic stress disorder: preliminary findings using magnetic resonance spectroscopy. Depress Anxiety. 2014;31:115-23.

163. Sartor CE, McCutcheon VV, Pommer NE, et al. Common genetic and environmental contributions to post-traumatic stress disorder and alcohol dependence in young women. Psychol Med. 2011;41:1497-505.

164. Saunders EC, Lambert-Harris C, McGovern MP, et al. The prevalence of posttraumatic stress disorder symptoms among addiction treatment patients with cocaine use disorders. J Psychoactive Drugs. 2015;47:42-50.
165. SchenkS, Newcombe D. Methylenedioxymethamphetamine (MDMA) in psychiatry: pros, cons, and suggestions. JClin Psychopharmacol.2018;38:632-8.

166. Schiele MA, Bandelow B, Baldwin DS, Pini S, Domschke K. A neurobiological framework of separation anxiety and related phenotypes. Eur Neuropsychopharmacol. 2020;33:45-57.

167. Schmahl C, Bohus M. Translational research issues in dissociation. In: Vermetten E, Dorahy MJ, Spiegel D, Hrsg. Traumatic dissociation. Neurobiology and treatment. Washington, DC/London: American Psychiatric Publishing; 2007. S. 121-38.

168. Schnurr PP. Understanding pathways from traumatic exposure to physical health. In: Schnyder U, Cloitre M, Hrsg. Evidence based treatments for trauma-related psychological disorders. A practical guide for clinicians. Switzerland: Springer;2015. S. 87-103.

169. Seedat S. Interventions to improve psychological functioning and health outcomes of HIV-infected individuals with a history of trauma or PTSD. Curr HIV/AIDS Rep. 2012;9:344-50.

170. Sharma S, Ressler KJ. Genomic updates in understanding PTSD. Prog Neuropsychopharmacol Biol Psychiatry. 2019;90:197-203.

171. Sheerin CM, Bountress KE, Meyers JL, et al. Shared molecular genetic risk of alcohol dependence and posttraumatic stress disorder (PTSD). Psychol Addict Behav. 2020; https:// doi.org/10.1037/adb0000568.

172. Shipherd JC, Stafford J, Tanner LR. Predicting alcohol and drug abuse in Persian Gulf War veterans: What role do PTSD symptoms play? Addict Behav. 2005;30:595-9.

173. Short J, Cram F, Roguski M, et al. Thinking differently: re-framing family violence responsiveness in the mental health and addictions health care context. Int J Mental Health Nurs. 2019;28:1206-16.

174. Simpson TL, Lehavot K, Petrakis IL. No wrong doors: findings from a critical review of behavioral randomized clinical trials for individuals with co-occurring alcohol/drug problems and posttraumatic stress disorder. Alcohol Clin Exp Res. 2017;41:681-702.

175. Smith GT, Cyders MA. Integrating affect and impulsivity: the role of positive and negative urgency in substance use risk. DrugAlcohol Depend. 2016;163:S3-S12.

176. Smith NDL, Cottler LB. The epidemiology of post-traumatic stress disorder and alcohol use disorder. Alcohol Res. 2018;39:113-20.

177. Solursh L. Combat addiction: overview of implications in symptom maintenance and treatment planning. J Trauma Stress. 1989;2:452-62.

178. Sommer JL, El-Gabalawy R, Contractor AA, et al. PTSD's risky behavior criterion: associated risky and unhealthy behaviors and psychiatric correlates in a nationally representative sample. JAnxiety Disord. 2020;73:102247.https:// doi.org/10.1016/j.janxdis.2020.102247.

179. Somohano VC, Rehder KL, Dingle T, et al. PTSD symptom clusters and craving differs by primary drug of choice.JDual Diagn. 2019;15:233-42.

180. Soyka M, Batra A, Heinz A, Moggi F, Walter M, Hrsg. Suchtmedizin. München: Elsevier/Urban \& Fischer; 2020.

181. Staiger PK, Melville F, Hides L, et al. Can emotion-focused coping help explain the link between posttraumatic stress disorder severity and triggers for aubstance use in young adults? J Subst Abuse Treat. 2009;36:220-6.

182. Strathearn L, Mertens CE, Mayes L, et al. Pathways relating the neurobiology of attachment to drug addiction. Front Psychiatry. 2019;10:737. https://doi.org/10.3389/ fpsyt.2019.00737. 
183. Straus E, Norman SB, Haller M, et al. Differences in protective factors among U.S. veterans with posttraumatic stress disorder, alcohol use disorder, and their comorbidity: results from the National Health and Resilience in Veterans Study. DrugAlcoholDepend. 2019;194:6-12.

184. Suh J, Ressler KJ. Common biological mechanisms of alcoholuse disorder and post-traumatic stress disorder.Alcohol Res. 2018;39:131-145.

185. Syed SA, Nemeroff CB. Early life stress, mood, and anxiety disorders. Chronic Stress (Thousand Oaks). 2017; https:// doi.org/10.1177/2470547017694461.

186. Teicher MH, Samson JA. Annual research review: enduring neurobiological effects of childhood abuse and neglect. JChild Psychol Psychiatry. 2016;57:241-66.

187. TeicherMH,SamsonJA,Anderson CM, OhashiK. The effects of childhoodmaltreatment on brain structure, function and connectivity. Nat Neurosci Rev. 2016;17:652-66.

188. Toftdahl NG, Nordentoft M, Hjorthøj C. Prevalence of substance use disorders in psychiatric patients: a nationwide Danish population-based study. Soc Psychiatry Psychiatr Epidemiol.2016;51:129-40.

189. Trucco EM, Madan B, Villar M. The impact of genes on adolescent substance use: a developmental perspective. Curr Addict Rep. 2019;6:522-31.

190. Tull MT, McDermott MJ, Gratz KL, et al. Cocaine-related attentional bias following trauma cue exposure among cocaine dependent in-patients with and without posttraumatic stress disorder. Addiction. 2011;106:1810-8.

191. Tull MT, Bardeen JR, DiLillo D, et al. A prospective investigation of emotion dysregulation as a moderator of the relation between posttraumatic stress symptoms and substance use severity. JAnxiety Disord. 2015;29:52-60.

192. Tull MT, McDermott MJ, Gratz KL. Marijuana dependence moderates the effect of posttraumatic stress disorder on trauma cue reactivity in substance dependent patients. DrugAlcoholDepend.2016;159:219-26.

193. Valentino RJ, Van Bockstaele E. Endogenous opioids: the downside of opposing stress. Neurobiol Stress. 2015;1:23-32.

194. Valverde O, Rodríguez-Árias M. Modulation of 3, 4-methylenedioxymethamphetamine effects by endocannabinoid system. Curr Pharm Des. 2013;19:7081-9.

195. Verplaetse TL, McKee SA, Petrakis IL. Pharmacotherapy for co-occurring alcohol use disorder and post-traumatic stress disorder:targeting theopioidergic, noradrenergic, serotonergic, and GABAergic/glutamatergic systems. Alcohol Res. 2018;39:193-205.

196. Vujanovic AA, Farris SG, BartlettBA, et al. Anxiety sensitivity in the association between posttraumatic stress and substance usse disorders: a systematic review. Clin Psychol Rev. 2018;62:37-55.

197. Vujanovic AA, Wardle MC, Smith LJ, Berenz EC. Reward functioning in posttraumatic stress and substance use disorders. Curr Opin Psychol. 2017;14:49-55.

198. Waldrop AE, Back SE, Verduin ML, Brady KT. Triggers for cocaine and alcohol use in the presence and absence of posttraumatic stress disorder. Addict Behav. 2007;32:634-9.

199. Walsh K, Elliott JC, Shmulewitz D, et al. Trauma exposure, posttraumatic stress disorder and risk for alcohol, nicotine, and marijuana dependence in Israel. Compr Psychiatry. 2014;55:621-30.
200. Walters H, Kosten TA. Early life stress and the propensity to develop addictive behaviors. Int J Dev Neurosci. 2019;78:156-69.

201. Walton JL, Raines AM, Cuccurullo LAJ, et al. The relationship between DSM-5 PTSD symptom clusters and alcohol misuse among military veterans. Am J Addict. 2018;27:23-8.

202. Wang SC, Chen YC, Lee CH, Cheng CM. Opioid addiction, genetic susceptibility, and medical treatments: a review. Int J Mol Sci. 2019;20:4294. https://doi.org/10.3390/ ijms20174294.

203. Weiss NH, Tull MT, Sullivan TP, et al. Posttraumatic stress disorder symptoms and risky behaviors among traumaexposed inpatients with substance dependence: the influence of negative and positive urgency. Drug Alcohol Depend. 2015;155:147-53.

204. Weiss NH, Schick MR, Contractor AA, Dixon-Gordon KL. Posttraumatic stress disorder and substance use: identifying the underlying role of difficulties regulating positive emotions. Addict Behav. 2019;96:119-26.

205. Weiss NH, Tull MT, Anestis MD, Gratz KL. The relative and unique contributions of emotion dysregulation and impulsivity to posttraumatic stress disorder among substance dependent inpatients. Drug Alcohol Depend. 2013;128:45-51.

206. Welch AE, Caramanica K, Maslow CB, et al. Trajectories of PTSD among lower Manhattan residents and area workers following the 2001 World Trade Center disaster, 2003-2012. J Trauma Stress. 2016;29:158-66.

207. Wemm SE, Sinha R. Drug-induced stress responses and addiction risk and relapse. Neurobiol Stress. 2019;10:100148. https://doi.org/10.1016/j.ynstr.2019.100148.

208. Wittenauer Welsh J, Knight JR, Shu-Yeu Hou S, et al. Association between substance use diagnoses and psychiatric disorders in an adolescent and young adult clinic-based population. JAdolesc Health. 2017;60:648-52.

209. Wolf EJ, Miller MW, Krueger RF, et al. Posttraumatic stress disorder and the genetic structure of comorbidity. JAbnorm Psychol. 2010;119:320-30.

210. Xian H, Chantarujikapong SI, Scherrer JF, et al. Genetic and environmental influences on posttraumatic stress disorder, alcohol and drug dependence in twin pairs. Drug Alcohol Depend. 2000;61:95-102.

211. Xian H, Scherrer JF, Grant JD, et al. Genetic and environmental contributions to nicotine, alcohol and cannabis dependence in male twins. Addiction. 2008;103:1391-8.

212. Yehuda R, Yang RK, Buchsbaum MS, Golier JA. Alterations in cortisol negative feeback inhibition as examined using the ATCH response to cortisol administraion in PTSD. Psychoneuroendocrinology. 2006;31:447-451.

213. Young RM. Harmful drinking in military veterans with posttraumatic stress disorder: association with the D2 dopamine receptor al allele. Alcohol Alcohol.2002;37:451-6.

214. Young LB, Timko C, Pulido RD. Traumatic childhood experiences and posttraumatic stress disorder among veterans in substance use disorder treatment. J Interpers Violence. 2020; https:// doi.org/10.1177/0886260519900937.

215. Zohar J, Yahalom H, Kozlovsky N, et al. High dose hydrocortisone immediately after trauma may alter the trajectory of PTSD: interplay between clinical and animal studies. Eur Neuropsychopharmacol.2011;21:796-809.

Hinweis des Verlags Der Verlag bleibt in Hinblick auf geografische Zuordnungen und Gebietsbezeichnungen in veröffentlichten Karten und Institutsadressen neutral. 